\title{
Clinical development of metabolic inhibitors for oncology
}

\author{
Kathryn M. Lemberg, ${ }^{1,2}$ Sadakatali S. Gori, ${ }^{1,3}$ Takashi Tsukamoto, ${ }^{1,3,4}$ Rana Rais, ${ }^{1,3,4}$ and Barbara S. Slusher ${ }^{1,2,3,4,5,6}$ \\ 1Johns Hopkins Drug Discovery, ${ }^{2}$ Department of Oncology and The Sidney Kimmel Comprehensive Cancer Center, ${ }^{3}$ Department of Neurology, ${ }^{4}$ Department of Pharmacology and Molecular Sciences, \\ ${ }^{5}$ Department of Medicine, and ${ }^{6}$ Department of Psychiatry and Behavioral Science, Johns Hopkins University School of Medicine, Baltimore, Maryland, USA.
}

\begin{abstract}
Metabolic inhibitors have been used in oncology for decades, dating back to antimetabolites developed in the 1940s. In the past 25 years, there has been increased recognition of metabolic derangements in tumor cells leading to a resurgence of interest in targeting metabolism. More recently there has been recognition that drugs targeting tumor metabolism also affect the often acidic, hypoxic, immunosuppressive tumor microenvironment (TME) and non-tumor cell populations within it, including immune cells. Here we review small-molecule metabolic inhibitors currently in clinical development for oncology applications. For each agent, we evaluate the preclinical studies demonstrating antitumor and TME effects and review ongoing clinical trials. The goal of this Review is to provide an overview of the landscape of metabolic inhibitors in clinical development for oncology.
\end{abstract}

\section{Introduction}

Tumor metabolism has been an area of basic science and clinical interest for many decades, dating to Otto Warburg's classic observations of high tumor glycolytic rates in the presence of oxygen (1). Metabolic demands of leukemia cells for purine and pyrimidine base production were first targeted with the development of aminopterin (2) and later methotrexate as some of the earliest antimetabolite therapies. In more recent years it has become widely accepted that reprogrammed energy metabolism of some tumor cells improves their ability to generate substrates needed for biomass (e.g., reducing equivalents, nucleotides) (3). Considerable efforts have also been made to understand the relationship between mutations in oncogenes/tumor suppressors and the resulting changes to metabolic pathways (4). Recent examples exist of successful translation of discovered cancer cell metabolic derangements into FDA-approved metabolic inhibitors. For example, D-2-hydroxyglutarate-producing (D2HG-producing) mutations in isocitrate dehydrogenase (IDH) were identified in sequencing efforts of gliomas and acute myeloid leukemia (AML) $(5,6)$. Detailed understanding of the metabolic and epigenetic consequences led to development and approval of ivosidenib and enasidenib for relapsed/refractory IDH-mutated AML (7). However, for many other observed metabolic derangements in tumor cells, clinical translation has been

Conflict of interest: RR and BSS are inventors on Johns Hopkins University patents covering novel glutamine antagonist prodrugs: "Prodrugs of glutamine analogs" (W02017023774A1); "Methods and compositions for treating metabolic reprogramming disorders" (W02017023787A1); "Novel glutamine antagonists and uses thereof" (W02019071110A1). These patents have been licensed to Dracen Pharmaceuticals Inc. RR and BSS are founders of and hold equity in Dracen Pharmaceuticals Inc. BSS also serves as a scientific consultant to Dracen. This arrangement has been reviewed and approved by the Johns Hopkins University in accordance with its conflict-of-interest policies. Copyright: (5) 2022, Lemberg et al. This is an open access article published under the terms of the Creative Commons Attribution 4.0 International License.

Reference information: J Clin Invest. 2022;132(1):e148550.

https://doi.org/10.1172/JCl148550. more challenging. Reasons for this hurdle include the flexibility of cancer metabolic pathways to circumvent points of inhibition, leading to insufficiency of monotherapy; overlap with metabolism of healthy cells, which narrows the therapeutic index; and the difficulty of accessing some tumors of interest - particularly CNS tumors or tumors within a dense environment of supporting cells.

The tumor microenvironment (TME) contains connective tissue, vessels, fibroblasts, and immune cell populations and plays a critical role in tumor growth, survival, and spread (4). Efforts have been made to target aspects of the TME in the treatment of cancer - for example with angiogenesis inhibitors (8), connective tissue antagonists (9), and immunomodulatory therapies. As the TME is often acidic, hypoxic, and nutritionally a suboptimal environment for immune surveillance and antitumor immune activity (10), there has been interest in characterizing the metabolic needs of antitumor immune cells and the effect of tumor metabolic inhibition on the greater TME. Several excellent recent reviews detail basic and early translational investigations into this area (11-13). Specifically, studies have focused on markers of immune susceptibility following metabolic inhibition and whether combination with immunotherapy may improve outcomes. In this Review we describe the current landscape of metabolic inhibitors in clinical development for oncology applications (Figure 1). These include agents with considerable clinical data (e.g., IDO1 inhibitors) as well as newer entries (e.g., sphingosine kinase-2 inhibitors). For each compound (Figure 2), we review available preclinical data (Table 1) and current clinical trials including novel combinations (Tables 2 and 3). The goal is to synthesize findings to date and motivate further translational research into the most effective ways to combine metabolic inhibition with chemotherapy and/or immunotherapy for improved treatment of cancer.

\section{Metabolic inhibitors in clinical trials}

Targeting IDO: second wave of clinical trials. Indoleamine 2,3-dioxygenase 1 (IDO1) converts tryptophan, which is required for $\mathrm{T}$ cell 


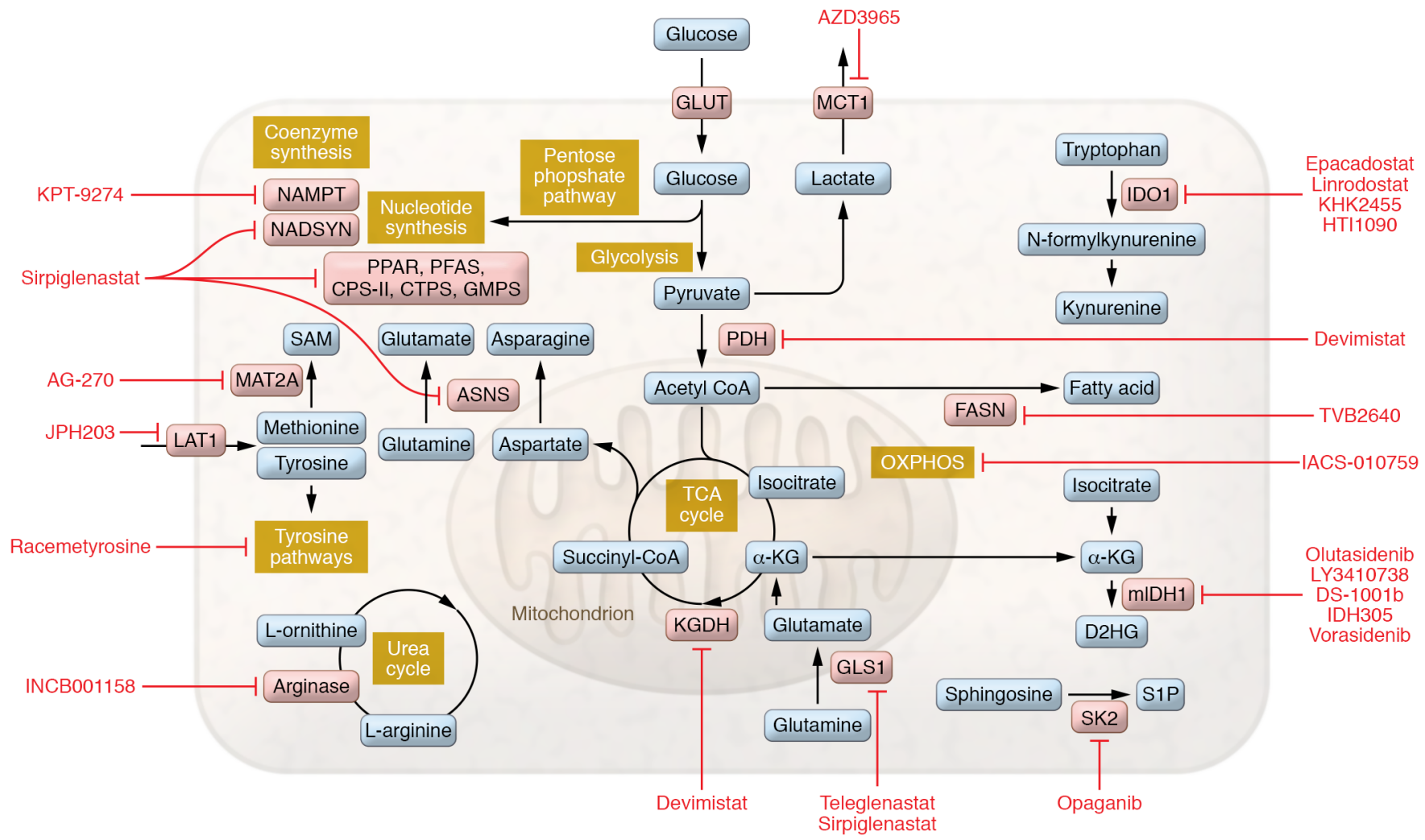

Figure 1. Metabolic inhibitors under clinical investigation for oncology applications. Schematic depicts the metabolic pathways and processes inhibited by agents described in this Review. Agents are shown in red text. Key enzymes are shown in pink. Pathways are labeled in yellow. Metabolites are shown in blue.

activity, into the immunosuppressive metabolite kynurenine. Accumulating evidence suggests that many tumors and immune cells within the TME increase their IDO1 activity as part of a strategy to decrease $\mathrm{T}$ cell responses and elude anticancer surveillance (14, 15). The mechanisms by which IDO1 adversely affects the immune system are multifold and include inhibition of local CD $8^{+} \mathrm{T}$ effector (Teff) cells; increasing formation of $\mathrm{CD} 4^{+} \mathrm{T}$ regulatory cells (Tregs) (16) and myeloid-derived suppressor cells (MDSCs) (17); inhibition of mTOR; and activation of the aryl hydrocarbon receptor $(18,19)$. IDO1 can also be induced in specific subsets of antigen-presenting cells, leading to immune tolerance to tumor antigens (20).

Given the therapeutic potential, multiple small-molecule IDO1 inhibitors have been developed, and were shown to restore antitumoral $\mathrm{T}$ cell immunity and synergize with immune checkpoint inhibitors in preclinical models (21). Based on these encouraging results, several companies took IDO1 inhibitors into clinical development (22). Although initial phase I/II trials encouraged the idea that IDO1 inhibition may improve responses to anti-PD-1 immune checkpoint therapy, the failed randomized phase III studies of epacadostat and navoximod in metastatic melanoma in combination with pembrolizumab led to an abrupt pause in IDO1 inhibitor development. Postmortem cautionary narratives followed, arguing that the initial failures may have been due to inadequately planned clinical trials designed with limited preclinical data, lack of confirmed target engagement, lack of a rationale for patient selection, need for improvements to patient stratification, and/or lack of biomarkers to guide dosing (23). Thus, enthusiasm for IDO1 inhibitors was not extinguished, and with revised clinical strategies, development of several inhibitors was restarted across a range of tumor types, including metastatic prostate (24), bladder $(25,26)$, glioblastoma (27), endometrial (28), hepatocellular (29), and head and neck squamous cell carcinoma (30), with most studies including combinations with immunotherapy.

There also have been new entrants into the IDO1 field, including KHK2455, which is being evaluated in advanced bladder cancer (31) in combination with anti-PD-L1. Some of the newer inhibitors, such as linrodostat and KHK2455, are reported to be different from earlier inhibitors as they inhibit IDO1 by competing with heme for binding to the apoenzyme (apo-IDO1) (32). This binding event prevents apo-IDO1 from forming an active complex, ensuring a durable inhibitory effect (33). Targeting strategies that account for both IDO1 and tryptophan 2,3-dioxygenase (TDO) have also been rationalized, and dual IDO1/TDO inhibitors (e.g., HTI-1090/SHR9146) were in clinical trials (34), though the evidence for this approach to date is indirect (35).

Different drug combinations with IDO1 inhibitors are also being attempted. While there is a mechanistic rationale for the combination with anti-PD-1, more efficacious combinations have been demonstrated with DNA-damaging chemotherapies (36) and cancer vaccines (37). With better rationalized compounds and improved trial designs, the verdict is still out on the utility of this class of immune-modulating drugs.

Targeting mutant IDH in the brain. Isocitrate dehydrogenases 1 and 2 (IDH1 and IDH2) catalyze the oxidative decarboxylation of isocitrate to $\alpha$-ketoglutarate $(\alpha-\mathrm{KG})$ in an NADP-dependent manner. These enzymes represent the most frequently mutated meta- 

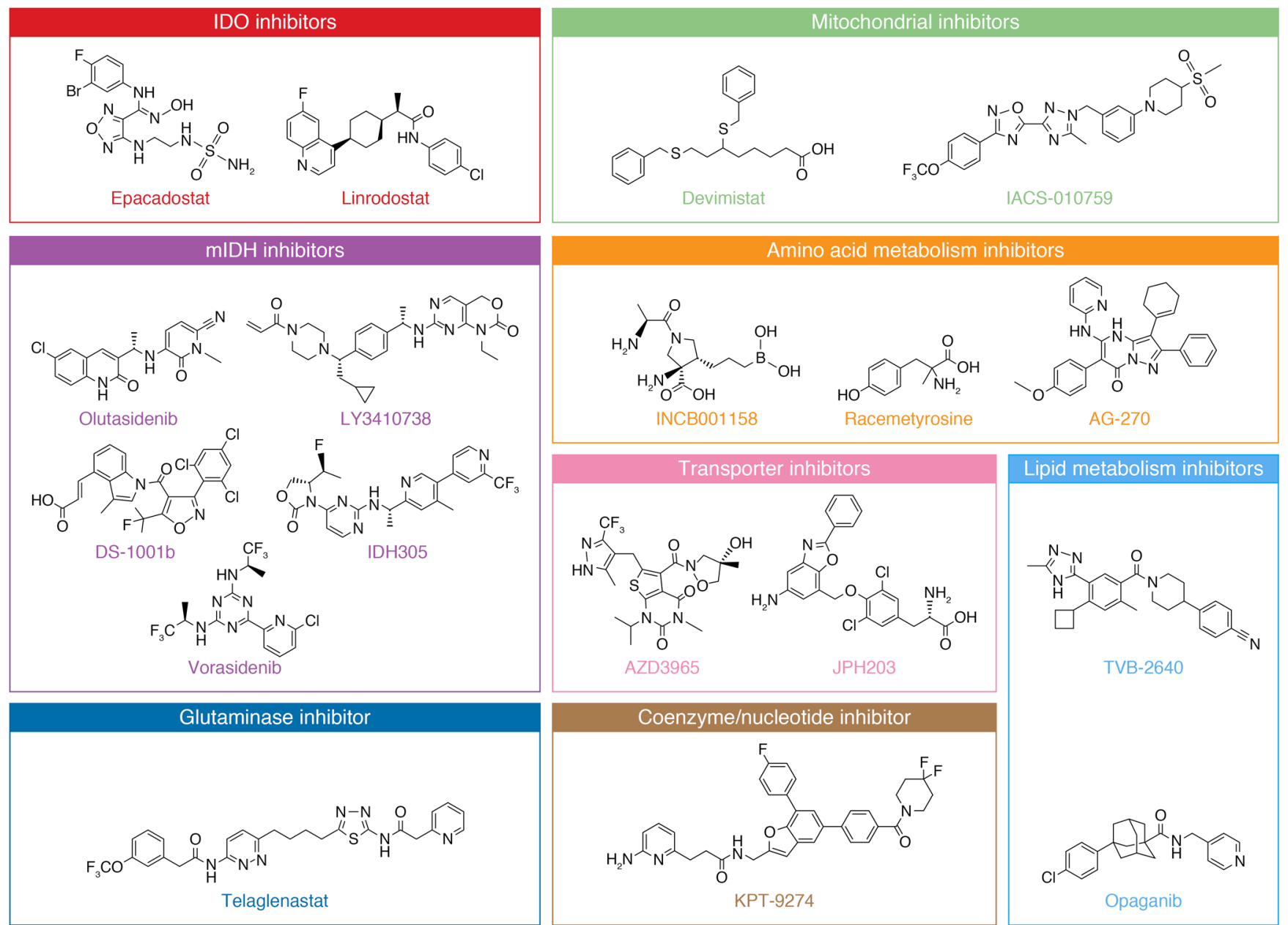

Figure 2. Structures of metabolic inhibitors currently under clinical investigation, grouped by target pathway. Structures of KHK2455, HTI-1090, and sirpiglenastat have not yet been disclosed.

bolic genes in human cancer (38). Mutations in IDH1 (at Arg132) and IDH2 (at Arg172) result in loss of the native catalytic activity but gain of a new catalytic function, namely, NADPH-dependent reduction of $\alpha$-KG to D2HG $(5,39)$. Elevated D2HG can induce epigenetic alterations thought to play a critical role in malignant progression (40). Furthermore, D2HG was reported to suppress activation of the classical and alternative complement pathways as well as $\mathrm{T}$ cell response, potentially promoting immune escape of IDH-mutant tumors (41). These carcinogenic features of D2HG make mutant forms of IDH attractive targets for therapeutic agents specific to IDH-mutant cancers. Ivosidenib (AG-120) and enasidenib (AG-221) are first-in-class inhibitors of mutant IDH1 and IDH2 (mIDH1 and mIDH2) $(42,43)$, respectively, which were recently approved by the FDA for the treatment of AML with IDH1 or IDH2 mutation.

Frequent IDH mutations in brain tumors have prompted interest in improving CNS penetration of mIDH inhibitors. Several second-generation brain-penetrant inhibitors have been developed, including LY3410738 (44), DS-1001b (45), olutasidenib (FT-2102; ref. 46), and vorasidenib (AG-881; ref. 47), and multiple trials are under way in patients with mIDH glioma (48-53). Because elevated D2HG can lead to genomic CpG hypermethylation, some of these inhibitors are being investigated in combination with DNA hypomethylating agents $(54,55)$. A recent preclinical study suggests that mIDH1 inhibition may improve survival when combined with anti-PD-L1 (56). Toward this end, olutasidenib combined with nivolumab is being evaluated in patients with advanced solid tumors and gliomas with mIDH1 (57).

Inhibition of glutaminase by telaglenastat (CB-839). Glutamine is a critical fuel for metabolic processes; it serves as a nitrogen and carbon source in biosynthetic pathways and is essential for energy generation as well as cellular homeostasis. Several cancers have been described as "glutamine-addicted" because of their exceptional metabolic demands.

Telaglenastat (CB-839) is a first-in-class, potent and selective inhibitor of the enzyme glutaminase (GLS1), which catalyzes the deamidation of glutamine to glutamate. Telaglenastat inhibits both splice variants of GLS1, the kidney type (KGA) and glutaminase C (GAC), with nanomolar potency (58). Glutaminolysis inhibition by telaglenastat has been shown to prime the tumor environment for immunological modulation, including enhancing the cytotoxic activity of tumor-infiltrating lymphocytes (TILs) $(59,60)$. Telaglenastat has shown efficacy in several preclinical cancer models that exhibit glutamine dependence, including triple-negative breast 
Table 1. Metabolic inhibitors in development: preclinical studies

\begin{tabular}{|c|c|c|c|c|}
\hline & Target & Preclinical efficacy models & TME/immune effect & Refs. \\
\hline $\begin{array}{l}\text { Epacadostat }{ }^{A} \\
(\text { INCB024360) }\end{array}$ & ID01 & $\begin{array}{l}\text { Syngeneic } \mathrm{CT} 26 \text { colon and PANO2 } \\
\text { pancreatic xenografts }\end{array}$ & $\begin{array}{l}\text { Promoted T/NK cell growth; reduced Tregs in coculture; } \\
\text { antitumor activity required lymphocytes }\end{array}$ & 170,171 \\
\hline $\begin{array}{l}\text { Linrodostat } \\
\text { (BMS-986205) }\end{array}$ & $\begin{array}{c}\text { IDO1 } \\
\text { (apo-enzyme) }\end{array}$ & Limited; PK/PD of plasma drug and tumor KYN & $\begin{array}{c}\text { Restored T cell proliferation in presence of IDO1- } \\
\text { expressing DCs }\end{array}$ & 172 \\
\hline КНК2455 & $\begin{array}{c}\text { IDO1 } \\
\text { (apo-enzyme) }\end{array}$ & Not disclosed & Not available & \\
\hline $\begin{array}{l}\text { HTI-1090 } \\
\text { (SHR9146) }\end{array}$ & ID01 and TDO & Not disclosed & Not available & \\
\hline $\begin{array}{l}\text { Olutasidenib } \\
\text { (FT-2102) }\end{array}$ & mIDH1 & Inhibition of D2HG in HCT116-IDH1-R132H/+ xenografts & Not available & 46 \\
\hline LY3410738 & mIDH1 (covalent) & $\begin{array}{l}\text { Inhibition of D2HG in orthotopic glioma; antitumor } \\
\text { efficacy in AML PDX }\end{array}$ & Not available & 44 \\
\hline DS-1001b & mIDH1 & Chondrosarcoma and GBM xenografts & Not available & 45,173 \\
\hline IDH305 & $\mathrm{mIDH} 1$ & IDH1-mutant melanoma & Not available & 174 \\
\hline $\begin{array}{l}\text { Vorasidenib } \\
\text { (AG-881) }\end{array}$ & mIDH1 and mIDH2 & $\begin{array}{l}\text { Inhibition of D2HG production in orthotopic glioma } \\
\text { model }\end{array}$ & Not available & 47 \\
\hline $\begin{array}{l}\text { Telaglenastat } \\
\text { (CB-839) }\end{array}$ & $\begin{array}{c}\text { GLS1 } \\
\text { (KGA and GAC) }\end{array}$ & TNBC, NSCLC, AML, RCC & With anti-PD-1 increased TILs in murine melanoma & $58,60-63$ \\
\hline $\begin{array}{l}\text { Sirpiglenastat } \\
\text { (DRP-104) }\end{array}$ & $\begin{array}{l}\text { All glutamine-utilizing } \\
\text { enzymes }\end{array}$ & Syngeneic colon cancer & $\begin{array}{c}\text { Increased tumor-infiltrating T cells, NK cells, TAMs; } \\
\text { decreased MDSCs }\end{array}$ & 86 \\
\hline AZD3965 & MCT1 and MCT2 & SCLC, lymphoma & Increased tumor DCs and NK cells & 91,93 \\
\hline $\begin{array}{l}\text { Devimistat } \\
\text { (CPI-613) }\end{array}$ & $\begin{array}{l}\text { Mitochondrial KGDH } \\
\text { and PDH }\end{array}$ & Pancreatic and NSCLC xenografts & $\begin{array}{l}\text { Increased B lymphocyte gene signature } \\
\text { in AML responders }\end{array}$ & $100,102,175,176$ \\
\hline IACS-010759 & Complex I/OXPHOS & Brain tumor, AML & $\begin{array}{l}\text { Decreased TME acidity; combination with anti-PD-1 } \\
\text { in NSCLC }\end{array}$ & $110-112,177$ \\
\hline $\begin{array}{l}\text { INCB001158 } \\
\text { (CB-1158) }\end{array}$ & Arginase I & Syngeneic melanoma, colon, breast xenografts & Increased tumor T cell infiltration & 120 \\
\hline $\begin{array}{l}\text { Racemetyrosine } \\
\text { (SM-88) }\end{array}$ & Tyrosine mimetic & HCT116 colon & Not available & 125 \\
\hline JPH2O3 & LAT1 & Colon, cholangiocarcinoma, thyroid & Reduced inflammation in autoimmune models & $132,133,178-180$ \\
\hline$A G-270$ & MAT2A & KP4 pancreatic, NSCLC, and esophageal SCC PDX & Not available & $137,138,181,182$ \\
\hline TVB-2640 & FAS & Not available & Not available & 144 \\
\hline $\begin{array}{l}\text { Opaganib } \\
\text { (ABC294640) }\end{array}$ & SK2 & Mammary adenocarcinoma & Not available & 150,183 \\
\hline КРТ-9274 & NAMPT/PAK4 & RMS, RCC & $\begin{array}{l}\text { Enriched tumor expression of IFN- } \alpha \text { and }-\gamma \text { response } \\
\text { and antigen processing pathways }\end{array}$ & 156,157 \\
\hline
\end{tabular}

${ }^{A}$ Some IDO inhibitors (e.g., epacadostat) are also agonists for the aryl hydrocarbon receptor, which may induce immunosuppression. AML, acute myeloid leukemia; D2HG, D-2-hydroxyglutarate; DC, dendritic cell; FAS, fatty acid synthase; GAC, glutaminase C; GBM, glioblastoma multiforme; GLS1, glutaminase; ID01, indoleamine 2,3-dioxygenase 1; KCA, kidney-type glutaminase; KGDH, ketoglutarate dehydrogenase; KYN, kynurenine; LAT1, large neutral amino acid transporter 1; MAT2A, methionine adenosyltransferase $2 \alpha ; \mathrm{MCT1} / 2$, monocarboxylate transporter 1 or 2; MDSC, myeloid-derived suppressor cell; mIDH1/2, mutant isocitrate dehydrogenase 1 or 2; NAMPT, nicotinamide phosphoribosyltransferase; NK, natural killer; NSCLC, non-small cell lung cancer; PDH, pyruvate dehydrogenase; PDX, patient-derived xenograft; PK/PD, pharmacokinetics/pharmacodynamics; RCC, renal cell carcinoma; RMS, rhabdomyosarcoma; SCC, squamous cell carcinoma; SCLC, small cell lung cancer; SK2, sphingosine kinase-2; TAM, tumor-associated macrophage; TDO, tryptophan 2,3-dioxygenase; TIL, tumor-infiltrating lymphocyte; TME, tumor microenvironment; TNBC, triple-negative breast cancer; Treg, regulatory T cell.

cancer (58), non-small cell lung cancer (NSCLC) (61), AML (62), renal cell carcinoma (RCC) $(63,64)$, and lymphoma models. Antiproliferative responses were shown to correlate with elevated GLS1 protein, particularly GAC $(58,65)$. These favorable data supported moving telaglenastat into clinical development.

Telaglenastat was well tolerated at daily doses greater than 1 $\mathrm{g}$ with some evidence of single-agent activity in relapsed/refractory leukemia patients (66). However, in general, the single-agent activity was modest, and subsequent efforts have focused on combination therapies. For example, a combination study with panitumumab and irinotecan is under way for treatment of metastatic and refractory RAS-wild-type colorectal cancer using PET/CT imaging biomarkers (67). Telaglenastat is also being investigated in metastatic RCC in combination with the multikinase inhibitor cabozantinib (68), as promising effects were observed preclinically and in a small expansion cohort of RCC patients in phase I trials (63). Early data from primary analysis of this trial show that the combination was well tolerated and the adverse events were consistent with known risks of both agents; however, the addition of telaglenastat did not improve the efficacy of cabozantinib in RCC. The median progression-free survival (PFS) was 9.2 months among patients treated with telaglenastat and cabozantinib, which 


\section{Table 2. Metabolic inhibitors in current clinical trials, part I}

\begin{tabular}{|c|c|c|c|c|}
\hline Agent & Combination agents & Phase & Tumor types & Ref. \\
\hline \multirow[t]{9}{*}{ Epacadostat ${ }^{\AA}$ (INCBO24360) } & Pembrolizumab & III & $\mathrm{RCC}$ & 184 \\
\hline & Tavo-EP (intratumoral IL-12), pembrolizumab & II & Unresectable head and neck SCC & 186 \\
\hline & Pembrolizumab & ॥ & Advanced sarcoma & 188 \\
\hline & INCMGA00012, bevacizumab, XRT & ॥ & Clioma/CBM & 189 \\
\hline & Retifanlimab & $\|$ & Urothelial carcinoma & 190 \\
\hline & XRT, intralesional SD101 & $1 / 11$ & Advanced solid tumors, lymphoma & 191 \\
\hline & DEC-205/NY-ESO-1 fusion protein CDX-1401; poly ICLC vaccine & $1 / 11$ & Ovarian, fallopian tube, or peritoneal cancer & 192 \\
\hline & SV-BR-1-GM vaccine, retifanlimab & $1 / 11$ & Breast cancer & 193 \\
\hline & Retifanlimab & $\|$ & Endometrial cancer & 194 \\
\hline \multirow[t]{8}{*}{ Linrodostat ${ }^{A}$ (BMS-986205) } & Nivolumab, temozolomide, XRT & 1 & Glioblastoma & 27 \\
\hline & Nivolumab & II & Endometrial adenocarcinoma and carcinosarcoma & 28 \\
\hline & Nivolumab & $1 / I I$ & Advanced solid tumors & 196 \\
\hline & Nivolumab, BCG & $\|$ & Bladder cancer & 25 \\
\hline & Relatlimab, nivolumab & $1 / 11$ & Advanced solid tumors & 197 \\
\hline & Nivolumab & II & Advanced gastric cancer & 198 \\
\hline & Nivolumab & ॥ & Advanced renal cell carcinoma & 199 \\
\hline & Nivolumab, gemcitabine, cisplatin & III & Invasive bladder cancer & 26 \\
\hline КНК2455 & Avelumab & 1 & Urothelial carcinoma & 31 \\
\hline HTI-1090 (SHR9146) & SHR-1210, apatinib & I & Solid tumors, metastatic cancer, neoplasms & 34 \\
\hline \multirow[t]{2}{*}{ Olutasidenib (FT-2102) } & Azacitidine, nivolumab, gemcitabine, cisplatin & $1 / 11$ & $\begin{array}{l}\text { Glioma, GBM, hepatobiliary tumors, cholangiocarcinoma, } \\
\text { chondrosarcoma, solid tumors with mIDH1 }\end{array}$ & 57 \\
\hline & Azacitidine, cytarabine & $1 / 11$ & AML, myelodysplastic syndrome & 200 \\
\hline
\end{tabular}

Only active trials as of May 18, 2021 are listed. ${ }^{A}$ Additional phase I trials exist, not listed due to space. Cy, cyclophosphamide; NA, not applicable; XRT, radiotherapy.

was similar to the 9.3 months observed with cabozantinib alone (69). Other combinations include carfilzomib (protease inhibitor) and dexamethasone for treating recurrent multiple myeloma (70); niraparib or talazoparib [poly(ADP-ribose) polymerase (PARP) inhibitors] for treating platinum-resistant BRCA-wild-type ovarian cancer (71); palbociclib (cyclin-dependent kinase inhibitor) for treating solid tumors (72); and sapanisertib (mTOR inhibitor) for treating advanced NSCLC (73).

In addition to combination studies, telaglenastat is also being explored in patients with specific tumor mutations. A phase II basket trial is evaluating telaglenastat in patients with tumors bearing NF1, KEAP1/NRF2, and STK11/LKB1 aberrations (74). KRAS-mutant tumors with functional inactivation of LKB1 and a KEAP1 co-mutation exhibit increased glutamine dependence to successfully adapt to oxidative and energetic stress. This metabol- ic requirement for glutaminolysis presents a therapeutic vulnerability in cancers with genetic, epigenetic, or post-transcriptional alterations in the KEAP1/NRF2 pathway (75). Another early-stage trial is exploring combinations with radiation therapy and temozolomide for IDH-mutated astrocytomas (76). Trials of telaglenastat in combination with osimertinib (kinase inhibitor) for treating EGFR-mutated stage IV NSCLC (77) and in combination with pembrolizumab/standard-of-care chemotherapy in patients with KEAP1/NRF2-mutated NSCLC are also under way (78).

Broad glutamine antagonism with sirpiglenastat (DRP-104). Sirpiglenastat (DRP-104) is a tumor-targeted prodrug of the glutamine antagonist 6-diazo-5-oxo-L-norleucine (DON), which was identified in the 1950 s as a potent anticancer agent (79). DON is a mechanism-based, irreversible inhibitor of all glutamine-utilizing enzymes (80), and thus broadly inhibits metabolic pathways that 


\section{Table 3. Metabolic inhibitors in current clinical trials, part II}

\begin{tabular}{|c|c|c|c|c|}
\hline Agent & Combination agents & Phase & Tumor types & Ref. \\
\hline \multirow[t]{12}{*}{ Telaglenastat (CB-839) } & Panitumumab, irinotecan & $\mathrm{I} / \mathrm{II}$ & CRC, RAS wild-type & 67 \\
\hline & Cabozantinib & $\|$ & $\mathrm{RCC}$ & 68 \\
\hline & NA & ॥ & NF1-MPNST, advanced solid tumors & 74 \\
\hline & Temozolomide, radiation & । & Anaplastic or diffuse astrocytoma, IDH-mutant & 76 \\
\hline & Osimertinib & $1 / 11$ & Advanced NSCLC & 77 \\
\hline & Carboplatin, pemetrexed, pembrolizumab & $\|$ & NSCLC with KEAP1, NRF2, NFE2L2 mutation & 78 \\
\hline & Carfilzomib, dexamethasone & I & Plasma cell myeloma & 70 \\
\hline & NA & I & BRCA-wild-type ovarian cancer & 71 \\
\hline & Palbociclib & $1 / 11$ & Solid tumors, NSCLC, CRC with KRAS mutations & 72 \\
\hline & Sapanisertib & । & Leptomeningeal neoplasms, NSCLC, brain cancer & 73 \\
\hline & Talazoparib & $\|$ & Prostate cancer & 203 \\
\hline & Azacitidine & $1 / 11$ & Myelodysplastic syndrome & 204 \\
\hline Sirpiglenastat (DRP-104) & Atezolizumab & $1 / 11$ & Advanced solid tumors, NSCLC, HNSCC & 90 \\
\hline AZD3965 & NA & $\|$ & Solid tumors, lymphoma & 95 \\
\hline \multirow[t]{8}{*}{ Devimistat $^{A}($ CPI-613) } & HD-AraC, mitoxantrone & III & AML & 109 \\
\hline & Bendamustine & $\|$ & T cell lymphoma; NHL & 205 \\
\hline & NA & $\|$ & Burkitt lymphoma/leukemia, B cell lymphoma & 206 \\
\hline & Oxaliplatin, irinotecan, 5-FU, leucovorin & $1 / 11$ & Pancreatic cancer & 207 \\
\hline & Cytarabine, mitoxantrone & $\|$ & AML, granulocytic sarcoma & 208 \\
\hline & Hydroxychloroquine & $1 / 11$ & Clear cell sarcoma & 209 \\
\hline & Gemcitabine, cisplatin & $1 / 11$ & Biliary tract cancer & 210 \\
\hline & Oxaliplatin, irinotecan, 5-FU, leucovorin & III & Metastatic pancreatic cancer & 108 \\
\hline IACS-010759 & NA & 1 & AML & 114 \\
\hline \multirow[t]{4}{*}{ INCB001158 (CB-1158) } & Pembrolizumab & $1 / 11$ & Advanced/metastatic solid tumors & 121 \\
\hline & Oxaliplatin, leucovorin, 5-FU, gemcitabine, cisplatin, paclitaxel & $1 / 11$ & Advanced solid tumors & 118 \\
\hline & Daratumumab & $1 / 11$ & Multiple myeloma & 211 \\
\hline & Retifanlimab & I & Advanced solid tumors & 212 \\
\hline \multirow[t]{5}{*}{ Racemetyrosine (SM-88) } & Gemcitabine, nab-paclitaxel, mFOLFIRINOX & III & Metastatic pancreatic cancer & 213 \\
\hline & NA & $1 / 11$ & Breast cancer & 214 \\
\hline & NA & $\|$ & Metastatic breast cancer, $\mathrm{HR}^{+}$breast carcinoma & 215 \\
\hline & Methoxsalen, phenytoin, sirolimus (MPS) & $\|/\|$ & Pancreatic cancer & 216 \\
\hline & MPS & $\|$ & Sarcoma, Ewing sarcoma & 217 \\
\hline JPH2O3 & NA & $\|$ & Advanced biliary tract cancer & 218 \\
\hline AG-270 & Docetaxel, nab-paclitaxel, gemcitabine & I & Advanced solid tumors, lymphoma & 219 \\
\hline \multirow[t]{4}{*}{ TVB-2640 } & Bevacizumab & $\|$ & Astrocytoma & 146 \\
\hline & NA & I & Colon cancer & 220 \\
\hline & Paclitaxel, trastuzumab & $\|$ & Advanced breast carcinoma, HER2 $2^{+}$breast carcinoma & 147 \\
\hline & NA & $\|$ & KRAS NSCLC & 148 \\
\hline \multirow[t]{2}{*}{ Opaganib (ABC294640) } & Hydroxychloroquine & $\|$ & Cholangiocarcinoma & 153 \\
\hline & Abiraterone, enzalutamide & $\|$ & Metastatic castration-resistant prostate cancer & 154 \\
\hline \multirow[t]{2}{*}{ KPT-9274 } & Niacin, nivolumab & I & Advanced cancers, NHL, melanoma & 160 \\
\hline & Niacin & I & Advanced cancers, NHL & 221 \\
\hline
\end{tabular}

Only active trials as of May 18, 2021 are listed. ${ }^{A}$ Additional phase I trials exist, not listed due to space. CRC, colorectal cancer; 5-FU, 5-fluorouracil; HD-AraC, high-dose cytarabine; HNSCC, head and neck squamous cell carcinoma; MPNST, malignant peripheral nerve sheath tumor; NHL, non-Hodgkin lymphoma.

require glutamine as a nutrient source. The main impediment in the clinical development of DON has been its dose-limiting toxicities to normal tissues (81), especially the gastrointestinal tract, which is highly glutamine dependent (82). By utilizing promoieties that are preferentially cleaved by tumor-enriched enzymes (83), sirpiglenastat is able to deliver DON preferentially to the tumor, increasing its therapeutic index.

Recent pioneering studies using an earlier glutamine antagonist prodrug, JHU083, discovered divergent metabolic pathways in tumor cells versus immune cells within the TME. While glutamine antagonism blocked oxidative and glycolytic metabolism in tumor cells, it made the TME less hypoxic, acidic, and nutrient-deprived, leading to marked upregulation of oxidative metabolism in the T cells, resulting in enhanced antitumor activity (84). JHU083 was also shown to markedly inhibit the generation and recruitment of MDSCs and increase inflammatory tumor-associated macrophages (TAMs) (85). This disentangled process has also been noted with sirpiglenastat, which significantly inhibits 
tumor growth with a tandem increase in TILs (including T cells, NK cells, and TAMs) and decreased MDSCs in mice with syngeneic MC38 tumors. Sirpiglenastat was found to be superior to anti-PD-1/PD-L1 monotherapy and showed antitumor synergy as a combination, resulting in long-term durable cures $(86,87)$. Sirpiglenastat also demonstrated promising effects in a glutaminedependent KRAS-mutant lung cancer model that carries KEAP1 mutations $(88,89)$ and reduced KEAP1-mutant tumor growth in both murine and patient-derived lung and squamous tumor models (88). These data suggest that sirpiglenastat may be a promising therapeutic agent in patients carrying such mutations.

Sirpiglenastat is currently being evaluated in phase I/IIa clinical trials as a single agent and in combination with atezolizum$\mathrm{ab}$ for the treatment of advanced solid tumors (90). Recently, sirpiglenastat received a fast-track designation for the treatment of advanced NSCLC patients whose tumors express mutations in KEAP1, NFE2L2, and/or STK11. A phase Ib trial in NSCLC patients is also planned to establish safety and tolerability in combination with pembrolizumab.

Lactate efflux inhibition with AZD3965. AZD3965 is a first-inclass, potent $\left(K_{I}=1.6 \mathrm{nM}\right)$, orally bioavailable, selective inhibitor of monocarboxylate transporter 1 (MCT1). As tumor cells show an increased dependence on glycolysis resulting in lactic acid production, they overexpress MCTs as a protective mechanism to efflux lactate and avoid intracellular acidification. Potent antiproliferative activity of AZD3965 has been demonstrated in multiple lymphoma cell lines (91) and in small cell lung cancer (SCLC) models (92). A pharmacodynamic biomarker study showed that AZD3965 therapy resulted in decreased lipid synthesis including decreased choline levels. The drop in choline was attributed to decreased choline kinase A levels following intracellular lactate accumulation (93). Studies also showed that AZD3965-treated tumors had an increased abundance of dendritic cells and NK cells. Another publication assessed proteomic perturbation in mammospheres isolated from ER-positive breast cancer cell lines (94) and found dramatic overexpression of mitochondrial proteins, implying that cancer stem cells may become resistant to stress by fortifying the capacity to produce ATP via oxidative mitochondrial metabolism. Currently AZD3965 is being evaluated in a phase I dose-ranging study (95). While limited data are available at this point, one published case report noted malignant hyperlactemic acidosis in a patient with metastatic melanoma following the first dose of AZD3965 (96). The patient, in a PET scan, showed high glucose uptake in the metastasized tumors with extensive disease burden while minimal uptake was observed in the brain, suggesting a Warburg effect-dominated lactate production and efflux from the tumors. However, off-target effects of MCT inhibition in key tissues such as liver and kidney by AZD3965 treatment presumably interfered with plasma clearance of lactate, thus precipitating the symptomatic deterioration.

Inhibition of mitochondrial metabolism with devimistat (CPI613) and IACS-010759. While appreciation of the Warburg effect has focused much of cancer metabolism research on aerobic glycolysis, mitochondrial metabolism has also been explored to target cancer. While efforts have generally focused on tumor cells, certain populations of immune cells, such as Tmem, Teff, and Treg cells, are also thought to rely on mitochondrial respiration and oxidative phosphorylation (OXPHOS) in the hypoxic TME (11, 97-99), but there have been limited reports on this activity with the current clinical agents, representing an area for exploration.

The mitochondrial inhibitor devimistat (CPI-613) is a lipoate analog that inhibits two lipoate-dependent enzymes, ketoglutarate dehydrogenase (KGDH) and pyruvate dehydrogenase (PDH), that control availability of substrates derived from glucose and glutamine for replenishing the citric acid cycle $(100,101)$. The mechanism of inhibition of these enzymes appears to be distinct: devimistat was shown to modulate KGDH in a redox-dependent manner (102), whereas phosphorylation is affected for PDH (100). Preclinical studies using devimistat showed single-agent activity in xenograft models for NSCLC and pancreatic tumors (100) and activity in combination with chloroquine in clear cell sarcoma (103). However, limited evidence exists from preclinical studies showing an effect of devimistat on the TME or specific immune cell populations. One recent study from a gastroadenocarcinoma model highlighted improved survival in deinnervated gastric cancer treated with devimistat in combination with the mTOR inhibitor RAD001, highlighting a possible role for nervous tissue in the TME of these tumors (104). As a single agent, devimistat has been evaluated in multiple phase I studies, including in combination with modified FOLFIRINOX (mFOLFIRINOX) for pancreatic cancer $(105,106)$ and in combination with cytarabine/mitoxantrone for relapsed/refractory AML. In pancreatic cancer, devimistat plus mFOLFIRINOX exhibited a $61 \%$ overall response rate (ORR) (105). In the AML study, there was an ORR of $50 \%$ and the drug appeared promising in older patients and those with poor risk cytogenetics (107). Pretreatment bone marrow samples from a subgroup of AML patient responders showed an increased B lymphocyte gene signature, suggesting a role for B cells in the activity of the combined treatment. Further work will be needed to investigate this observation and identify whether a potential clinical role for devimistat in combination with immune checkpoint inhibitors exists. Current phase II/III clinical trials of devimistat include combinations with mFOLFIRINOX (108) in metastatic pancreatic cancer and with cytarabine/mitoxantrone in older adults with relapsed/refractory AML (109).

A mitochondrial complex I inhibitor, IACS-010759, is also in clinical oncology trials (110). IACS-010759 inhibits growth and induces apoptotic cell death in tumor models thought to be reliant on OXPHOS. In AML cells, metabolic changes following IACS010759 treatment were thought to be due to decreased availability of substrates to fuel nucleotide biosynthesis (e.g., aspartate) (110). In a mouse model of melanoma brain metastases, IACS-010759 treatment decreased markers of tumor hypoxia (111). Consistent with this finding, in patient samples of melanoma brain metastases, gene expression data suggested suppression of immune cell genes and upregulation of OXPHOS genes, particularly in comparison with matched primary melanoma and lung metastases (111). In a PD-1-resistant NSCLC model, combination treatment with anti-PD-1 plus IACS-010759 improved treatment response compared with single agent (112). Human trials are in an early stage: partial results of a completed phase I study in advanced solid tumors have been reported (113), with an additional phase I trial ongoing in AML (114). Urine lactate is being explored as a biomarker of activity. 
Targeting of arginine metabolism with INCBO01158 (CB-1158). Several studies have investigated modulation of the amino acid arginine as an antitumor approach. Interactions between tumor cell arginine synthesis and pyrimidine metabolism have been shown to promote tumor growth $(115,116)$. Myeloid and Treg populations in the TME express high levels of arginase I, which can hydrolyze arginine and disrupt T cell expansion in the TME (117). The small molecule INCB001158 (CB-1158) was developed to inhibit arginase I and increase arginine availability. This agent was tested in a phase I/II clinical study in combination with gemcitabine for advanced biliary cancers (118) with median PFS 8.5 months (119). A recent study showed that myeloid cells expressing arginase I suppress $\mathrm{T}$ cell proliferation in vitro, and this could be reversed by INCBO01158 (120). Tumor xenograft models treated with INCB001158 showed decreased growth and increased $\mathrm{T}$ cell infiltration (120). A phase I clinical trial of INCBO01158 as a single agent and in combination with anti-PD-1 in patients with solid tumors is ongoing (121); preliminary data suggested that the orally available drug is well tolerated (122).

Mimicking tyrosine with racemetyrosine (SM-88). Racemetyrosine (SM-88) is a tyrosine mimetic being developed for the treatment of various cancers, including pancreatic, lung, breast, prostate, sarcoma, and lymphoma (123). Some cancer cells more avidly take up and consume tyrosine compared with normal cells. Racemetyrosine takes advantage of this difference and acts as a dysfunctional tyrosine to interrupt tyrosine-mediated metabolic pathways, including protein synthesis. Specifically, racemetyrosine is thought to disrupt synthesis of cancer cell mucin-1 protein, leading to increased oxidative stress (124), cell death, and enhanced immunogenicity. In some cell lines, the methyl ester of racemetyrosine (SM-88 ME) was found to increase ROS in a dose-dependent manner (125). In a mouse colon cancer xenograft model, racemetyrosine was reported to reduce HCT116 tumor growth (125). A first-in-human, open-label, pilot study of racemetyrosine was conducted in advanced metastatic cancer patients in combination with melanin, phenytoin, and sirolimus (SMK therapy) (123). In addition, a phase Ib/II, open-label, dose-escalation study of racemetyrosine was conducted in combination with methoxsalen, phenytoin, and sirolimus in patients with non-metastatic biochemically recurrent prostate cancer (126). Although the outcomes of these open-label studies appear encouraging (e.g., no serious adverse events) $(127,128)$, more definitive conclusions await placebo-controlled randomized clinical trials.

Inhibition of LAT1 with JPH2O3. JPH2O3 is a selective inhibitor of large neutral amino acid transporter 1 (LAT1), a heterodimeric transporter composed of a heavy subunit protein, 4F2hc, encoded by the $S L C 3 A 2$ gene, and a light subunit protein encoded by the SLC7A5 gene (129). LAT1 preferentially transports branchedchain amino acids (valine, leucine, isoleucine) and aromatic amino acids (tryptophan, tyrosine). LAT1 is overexpressed in many cancer cells, which consume these amino acids to sustain proliferation. Indeed, it was reported that overall survival and PFS of RCC patients were shorter in patients with high LAT1 versus those with low LAT1 expression (130). Because slowly dividing normal cells can rely on LAT2 for amino acid uptake (131), selective inhibition of LAT1 by JPH2O3 is believed to be cytotoxic to cancer cells while sparing normal cells. JPH2O3 showed antitumor activity in pre- clinical models of colon cancer (132), cholangiocarcinoma (133), and thyroid carcinoma (133). Preclinical data suggest that LAT1 inhibition may also disrupt uptake of citrulline, a precursor of arginine, by activated $\mathrm{T}$ cells, resulting in immunosuppressive effects by arginine deprivation (134).

A first-in-human open-label phase I study of JPH2O3 was conducted in Japanese patients with advanced solid tumors (135); a partial response was reported in one patient. The study indicated that the safety and efficacy of JPH2O3 could be predicted by genetic variants in the NAT2 gene, which encodes an $\mathrm{N}$-acetyltransferase responsible for phase II metabolism of JPH2O3. The study also suggested that plasma free amino acid levels and BMI are useful predictors of JPH2O3 efficacy (136). Randomized controlled phase II studies in patients with advanced biliary tract cancer are under way in Japan (University Hospital Medical Information Network [UMIN] Clinical Trials Registry UMINO00034080).

Inhibition of MAT2A with AG-270. Efforts have been made to target methionine metabolism in tumors with deletion of methylthioadenosine phosphorylase (MTAP) (137), which is often co-deleted with the tumor suppressor CDKN2A. Deletion of MTAP results in accumulation of its substrate, methylthioadenosine, which has been shown to be an endogenous inhibitor of protein arginine methyltransferase 5 (PRMT5). The PRMT5-catalyzed enzymatic process can be further downregulated by starving the enzyme of its substrate, $S$-adenosyl methionine (SAM), via inhibition of methionine adenosyltransferase $2 \alpha$ (MAT2A). Because PRMT5 plays a critical role in the pathological progression of several human cancers, MAT2A inhibition is considered synthetically lethal with MTAP deletion (138).

AG-270 is an orally active inhibitor of MAT2A, which dosedependently reduced SAM levels in tumor and blocked tumor growth in a pancreatic xenograft model (137). The preliminary results from an ongoing, first-in-human, phase I trial of AG-270 in patients with advanced solid tumors with homozygous deletion of MTAP showed reduction in plasma SAM across a range of doses, demonstrating target engagement (139). The next step in this phase I study is to evaluate AG-270 in combination with taxanebased chemotherapy, which demonstrated synergistic antiproliferative effects in preclinical MTAP-null tumor models (138).

Inhibition of fatty acid synthesis with TVB-2640. Fatty acid synthase (FAS), encoded by the FASN gene, possesses multiple catalytic domains, which participate in the biosynthesis of longchain fatty acids, mainly palmitic acid, from acetyl-CoA and malonyl-CoA. Since FAS was identified as a marker of poor prognosis in breast cancer patients (140), it has gained considerable attention as a therapeutic target. TVB-2640 is the most advanced of the FAS inhibitors reported to date. Although limited information is available on TVB-2640 (141, 142), its close analog, TVB-3166, was found to induce apoptosis in tumor cells and inhibit xenograft tumor growth as monotherapy (143). It was also reported to enhance the antitumor activity of taxanes through inhibition of tubulin palmitoylation and disruption of microtubule organization (144). A first-in-human study of TVB-2640 alone and with a taxane in advanced tumors demonstrated a manageable safety profile and potent target engagement (FAS inhibition) (145). TVB-2640 is now being studied in several phase II trials, including in patients with first relapse of high-grade astrocytoma in 
combination with bevacizumab (146); in combination with paclitaxel and trastuzumab in patients with HER2-positive metastatic breast cancer resistant to trastuzumab and taxane-based therapy (147); and in patients with KRAS-mutant NSCLC (148). It was recently reported that FAS contributes to functional maturation of Tregs and that loss of FASN from Tregs inhibits tumor growth, suggesting the therapeutic potential of this class of inhibitors in cancer immunotherapy (149).

Alteration of bioactive lipids with opaganib (ABC294640). Lipid metabolism and the effect of bioactive lipids on tumor cells and the TME are an active area of research and therapeutics development. Opaganib (ABC294640) is a sphingosine kinase-2 (SK2) inhibitor that reduces formation of the pro-proliferative, prosurvival lipid sphingosine-1-phosphate (S1P) (150). Opaganib suppressed proliferation of multiple tumor cells in vitro and reduced tumor growth in mice with mammary adenocarcinoma xenografts. Given wide interest in sphingolipids in inflammation, there are multiple preclinical studies showing immune-modulating effects of opagan$\mathrm{ib}$, though not in oncology models. These include an LPS model of inflammation in which targeting SK2 with opaganib increased macrophage inflammatory cytokine production (151). In a phase I study, opaganib was found to decrease plasma S1P levels as a marker of target engagement (152). Phase II studies in cholangiocarcinoma in combination with hydroxychloroquine (153) and in metastatic castration-resistant prostate cancer (154) are ongoing.

NAD salvage inhibition with KPT-9274. Many cancer cells upregulate nicotinamide phosphoribosyltransferase (NAMPT), the rate-limiting step for $\mathrm{NAD}^{+}$salvage; thus efforts have been made to target this pathway with NAMPT inhibitors (155). KPT9274 is a dual p21-activated kinase 4 (PAK4)/NAMPT inhibitor (156). It reduces the $\mathrm{NAD}^{+} / \mathrm{NADH}$ ratio in tumor cells and inhibits tumor growth in mouse models of sarcomas (156) and RCC (157). Interestingly, in KPT-9274-treated rhabdomyosarcoma xenografts, tumors showed transcriptomic enrichment in pathways of adaptive immunity, IFN- $\alpha$ and $-\gamma$ response, and antigen processing (156). Using another NAMPT inhibitor (GMX1778) targeted to murine glioblastoma multiforme by microparticles, investigators found increased markers of TILs and increased surface expression of PD-L1. In this model there was a robust survival advantage in animals treated with immune checkpoint inhibitor plus NAMPT inhibitor (158). A phase I study of KPT-9274 was recently completed (159), and a study of KPT-9274 in combination with nivolumab for melanoma was enrolling patients but recently terminated (160).

\section{Summary}

The breadth of metabolic pathways being targeted in oncology with new clinical compounds is evidence of how much has been learned about tumor metabolism from basic and translational research studies over the past few decades. Several established targets have multiple clinical compounds in later stages of development across a variety of tumor types (e.g., IDO1, IDH1), while many earlier-phase studies are being initiated with firstin-class agents targeting metabolic nodes not yet explored (e.g., IACS-010759, opaganib). Considering the critical role played by various metabolic pathways in normal cellular homeostasis, one major challenge in developing metabolic inhibitors for oncology lies in achieving antitumor activity without substantial impact on non-cancer cells. Some of the investigational drugs described above have been developed using strategies to improve the therapeutic index. For instance, as mentioned earlier, inhibition of glutamine metabolism by DON is toxic to the gastrointestinal tract, which is enriched with highly glutamine-consuming cells (82). As demonstrated by sirpiglenastat, however, the prodrug approach exploited for tumor-targeted delivery of DON appears to be effective in minimizing gastric toxicity. Furthermore, inhibition of neomorphic functions of mutant enzymes offers a unique opportunity to develop anticancer agents with a greater therapeutic index as demonstrated by the selective inhibition of mIDH1 and mIDH2.

One of the advantages unique to metabolic inhibitors is that biomarkers measurable in plasma can be found among substrates and products of target enzymes. Indeed, available biomarkers played a crucial role in the development of many of the metabolic inhibitors reviewed in this article. Measurements of D2HG particularly in relation to serum drug pharmacokinetics were used in the development of IDH1 inhibitors (46). Tumor and plasma levels of SAM, a product of the MAT2A-catalyzed reaction of methionine and ATP, were used as biomarkers of AG-270 target engagement in mouse xenografts (133) and a first-in-human trial (139), respectively, providing guidance for dose selection in future clinical studies. Similarly, urine lactate is being used as a biomarker in the trial of IACS-010759 after being found to be elevated in preclinical studies (110). Finally, as described above, imaging biomarkers using nuclear medicine tracers have been incorporated into clinical trials of telaglenastat (161). Given the remarkable technological advances in recent years in the areas of bioanalysis and metabolic profiling, we expect metabolic biomarkers will play an even larger role in future development.

At the same time, a challenge for development of metabolic inhibitors in oncology is in identifying the patients whose tumors are most likely to benefit from a given agent. Lack of or incomplete understanding of biomarkers for patient enrollment has been cited as a contributing factor for the negative results in clinical studies of metabolic inhibitors, including the ECHO-301 trial combining IDO1 inhibitor with immunotherapy (23). On the other hand, several strategies to identify patients most likely to respond have been used successfully with other metabolic inhibitors. One strategy is to make use of genetic markers found in tumor cells. This is useful when an identified gene mutation can be associated with the metabolic target of interest; this strategy was used in trials of the IDH inhibitors ivosidenib and enasidenib (7). Deletion of MTAP is being used for patient selection in trials of AG-270 (ClinicalTrials.gov NCT03435250). For other metabolic inhibitors, such as the glutaminase inhibitor telaglenastat, surrogate genetic biomarkers leading to tumor glutamine dependence are being employed (e.g., NRF2/KEAP1) (75). A second potential patient selection strategy includes the use of imaging markers to evaluate tumor metabolic pathways and follow early response to therapy. As an example, hyperpolarized ${ }^{13} \mathrm{C}$-pyruvate MRI has been identified as a noninvasive way of evaluating prostate cancer, where conversion from ${ }^{13} \mathrm{C}$-pyruvate to ${ }^{13} \mathrm{C}$-lactate is measured and used to identify tumor tissue and treatment response with greater sensitivity than conventional ${ }^{1} \mathrm{H}-\mathrm{MRI}$ techniques $(162,163)$. Hyperpolarized ${ }^{13} \mathrm{C}$-pyruvate-to-lactate flux has been associated with higher MCT-expressing tumors, raising the possibility of pairing 
this companion imaging technology with clinical trials of MCT inhibitors. With additional probes and technology innovations in active development (164), hyperpolarized MRI and other forms of metabolic imaging will be useful additions for clinical oncology patient selection and follow-up in future trials.

An area of focus in this Review was to describe the effects of metabolic inhibitors on the TME in preclinical studies and understand how these findings were translated to clinical studies. For several of the agents highlighted, however, the effect on the TME was not well explored. Additionally, many published preclinical studies used nonclinical compounds, which can present a challenge in directly interpreting the results for a clinical agent. For example, olutasidenib is currently in clinical trials in combination with nivolumab for advanced solid tumors and glioma (57), but published literature on use of an IDH inhibitor with immunotherapy used a nonclinical tool inhibitor, which showed limited efficacy (56). It will be of interest to learn whether the preclinical data collected with clinical candidates are available when in-human study results are published. In addition, as preclinical oncology models improve to better reflect the TME of human tumors (e.g., humanized rodent models), we anticipate it will be more straightforward to investigate the in vivo effects of metabolic inhibitors on the TME preclinically.

It is worth noting that additional classes of metabolic agents in development exist outside the scope of this Review, including small-molecule receptor blockers with consequences for metabol- ic processes (e.g., trigluazole; ref. 165), antibody-based therapies (e.g., anti-CD73; ref. 166), enzyme-based metabolic inhibitors (e.g., ADI-PEG-20; ref. 167), and metabolic inhibitors already developed for other diseases now under investigation in cancer (e.g., eflornithine; ref. 168). Metabolic manipulations for ex vivo priming of immune cells directed at cancer are an additional fascinating area of research, which has been discussed elsewhere (169).

While multiple strong reviews in the literature cover the basic and translational processes leading to development of metabolic inhibitors, this Review offers a unique perspective by focusing on those agents that have progressed to human clinical studies in oncology. With significant drug development efforts in this area, it is likely that with the right combination with chemo- and/or immunotherapy, several of the metabolic inhibitors outlined in this Review will be widely employed in clinical oncology in the future.

\section{Acknowledgments}

BSS, RR, TT, and SSG are supported by NIH grants R01CA193895, R01CA229451, and R01NS103927 and the Bloomberg Kimmel Institute for Cancer Immunotherapy. BSS is also supported by NIH grant R01CA226765. KML is supported by a CureSearch for Children's Cancer Young Investigator Award.

Address correspondence to: Barbara S. Slusher, Johns Hopkins School of Medicine, 855 North Wolfe Street, Baltimore, Maryland 21205 USA. Phone: 410.960.6162; Email: bslusher@jhmi.edu.
1. Koppenol WH, et al. Otto Warburg's contributions to current concepts of cancer metabolism. Nat Rev Cancer. 2011;11(5):325-337.

2. Farber $\mathrm{S}$, et al. Temporary remissions in acute leukemia in children produced by folic acid antagonist, 4-aminopteroyl-glutamic acid (Aminopterin). N Engl J Med. 1948;238(23):787-793.

3. Vander Heiden MG, et al. Understanding the Warburg effect: the metabolic requirements of cell proliferation. Science. 2009;324(5930):1029-1033.

4. Hanahan D, Weinberg RA. Hallmarks of cancer: the next generation. Cell. 2011;144(5):646-674.

5 . Ward PS, et al. The common feature of leukemia-associated IDH1 and IDH2 mutations is a neomorphic enzyme activity converting alpha-ketoglutarate to 2-hydroxyglutarate. Cancer Cell. 2010;17(3):225-234.

6. Parsons DW, et al. An integrated genomic analysis of human glioblastoma multiforme. Science. 2008;321(5897):1807-1812.

7. Golub D, et al. Mutant isocitrate dehydrogenase inhibitors as targeted cancer therapeutics. Front Oncol. 2019;9:417.

8. Garcia J, et al. Bevacizumab (Avastin ${ }^{\circledR}$ ) in cancer treatment: a review of 15 years of clinical experience and future outlook. Cancer Treat Rev. 2020;86:102017.

9. Van Cutsem E, et al. Randomized phase III trial of pegvorhyaluronidase Alfa with Nab-paclitaxel plus gemcitabine for patients with hyaluronan-high metastatic pancreatic adenocarcinoma. JClin Oncol. 2020;38(27):3185-3194.

10. Augustin RC, et al. Characteristics of the tumor microenvironment that influence immune cell functions: hypoxia, oxidative stress, metabolic alterations. Cancers (Basel). 2020;12(12):3802.

11. Leone RD, Powell JD. Metabolism of immune cells in cancer. Nat Rev Cancer. 2020;20(9):516-531.

12. Li X, et al. Navigating metabolic pathways to enhance antitumour immunity and immunotherapy. Nat Rev Clin Oncol. 2019;16(7):425-441.

13. Wolpaw AJ, Dang CV. Exploiting metabolic vulnerabilities of cancer with precision and accuracy. Trends Cell Biol. 2018;28(3):201-212.

14. Prendergast GC, et al. Discovery of IDO1 inhibitors: from bench to bedside. Cancer Res. 2017;77(24):6795-6811.

15. Theate I, et al. Extensive profiling of the expression of the indoleamine 2,3-dioxygenase 1 protein in normal and tumoral human tissues. Cancer Immunol Res. 2015;3(2):161-172.

16. Hippen $\mathrm{KL}$, et al. In vitro induction of human regulatory $\mathrm{T}$ cells using conditions of low tryptophan plus kynurenines. Am J Transplant. 2017;17(12):3098-3113.

17. Li A, et al. IDO1 inhibition overcomes radiationinduced "rebound immune suppression" by reducing numbers of IDO1-expressing myeloid-derived suppressor cells in the tumor microenvironment. Int J Radiat Oncol Biol Phys. 2019;104(4):903-912.

18. Prendergast GC, et al. Inflammatory reprogramming with IDO1 inhibitors: turning immunologically unresponsive 'cold' tumors 'hot'. Trends Cancer. 2018;4(1):38-58.

19. Brincks EL, et al. Indoximod opposes the immunosuppressive effects mediated by IDO and TDO via modulation of AhR function and activation of mTORC1. Oncotarget. 2020;11(25):2438-2461.
20. Munn DH, Mellor AL. IDO in the tumor microenvironment: inflammation, counter-regulation, and tolerance. Trends Immunol. 2016;37(3):193-207.

21. Prendergast GC, et al. Indoleamine 2,3-dioxygenase and its therapeutic inhibition in cancer. Int Rev Cell Mol Biol. 2018;336:175-203.

22. Platten M, et al. Tryptophan metabolism as a common therapeutic target in cancer, neurodegeneration and beyond. Nat Rev Drug Discov. 2019;18(5):379-401.

23. Van den Eynde BJ, et al. Is there a clinical future for IDO1 Inhibitors after the failure of epacadostat in melanoma? Annu Rev Cancer Biol. 2020;4(1):241-256.

24. Phase I/II study of immunotherapy combination BN-brachyury vaccine, M7824, ALT-803 and epacadostat (QuEST1). https://ClinicalTrials. gov/show/NCT03493945. Updated September 18, 2021. Accessed September 22, 2021.

25 . A study of nivolumab or nivolumab plus experimental medication BMS-986205 with or without Bacillus Calumette-Guerin (BCG) in BCG unresponsive bladder cancer that has not invaded into the muscle wall of the bladder. https://ClinicalTrials.gov/show/NCT03519256. Updated July 22, 2021. Accessed September 22, 2021.

26. A study of chemo only versus chemo plus nivo with or without BMS-986205, followed by post-surgery therapy with nivo or nivo and BMS986205 in patients with MIBC. https://ClinicalTrials.gov/show/NCT03661320. Updated August 25, 2021. Accessed September 22, 2021.

27. Nivolumab, BMS-986205, and radiation therapy with or without temozolomide in treating patients with newly diagnosed glioblastoma. https://Clin- 
icalTrials.gov/show/NCT04047706. Updated July 2, 2020. Accessed September 22, 2021.

28. Study of BMS-986205 and nivolumab in endometrial cancer or endometrial carcinosarcoma that has not responded to treatment. https://ClinicalTrials.gov/show/NCT04106414. Updated November 17, 2020. Accessed September 22, 2021.

29. BMS-986205 and nivolumab as first or second line therapy in treating patients with liver cancer. https://ClinicalTrials.gov/show/ NCT03695250. Updated June 29, 2021. Accessed September 22, 2021.

30. Nivolumab and BMS 986205 in treating patients with stage II-IV squamous cell cancer of the head and neck. https://ClinicalTrials.gov/show/ NCT03854032. Updated September 1, 2021. Accessed September 22, 2021.

31. KHK2455 (IDO inhibitor) plus avelumab in adult subjects with advanced bladder cancer. https:// ClinicalTrials.gov/show/NCT03915405. Updated April 30, 2021. Accessed September 22, 2021.

32. Nelp MT, et al. Immune-modulating enzyme indoleamine 2,3-dioxygenase is effectively inhibited by targeting its apo-form. Proc Natl Acad Sci U S A. 2018;115(13):3249-3254.

33. Ortiz-Meoz RF, et al. Characterization of apoform selective inhibition of indoleamine 2,3-dioxygenase. Chembiochem. 2021;22(3):516-522.

34. Phase I study of SHR9146 + SHR-1210 +/- apatinib in patients with advanced solid tumors. https:// ClinicalTrials.gov/show/NCT03491631. Updated June 6, 2021. Accessed September 22, 2021.

35. Du L, et al. Both IDO1 and TDO contribute to the malignancy of gliomas via the Kyn-AhR-AQP4 signaling pathway. Signal Transduct Target Ther. 2020;5(1):10.

36. Huang $\mathrm{H}$, et al. Nanoenabled reversal of IDO1-mediated immunosuppression synergizes with immunogenic chemotherapy for improved cancer therapy. Nano Lett. 2019;19(8):5356-5365.

37. Blair $\mathrm{AB}$, et al. IDO1 inhibition potentiates vaccine-induced immunity against pancreatic adenocarcinoma. JClin Invest. 2019;129(4):1742-1755.

38. Wang HY, et al. The comparison of clinical and biological characteristics between IDH1 and IDH2 mutations in gliomas. J Exp Clin Cancer Res. 2016;35:86.

39. Dang L, et al. Cancer-associated IDH1 mutations produce 2-hydroxyglutarate. Nature. 2009;462(7274):739-744.

40. Duncan CG, et al. A heterozygous IDH1R132H/ WT mutation induces genome-wide alterations in DNA methylation. Genome Res. 2012;22(12):2339-2355.

41. Zhang L, et al. D-2-hydroxyglutarate is an intercellular mediator in IDH-mutant gliomas inhibiting complement and T cells. Clin Cancer Res. 2018;24(21):5381-5391.

42. Popovici-Muller J, et al. Discovery of AG-120 (ivosidenib): a first-in-class mutant IDH1 inhibitor for the treatment of IDH1 mutant cancers. ACS Med Chem Lett. 2018;9(4):300-305.

43. Yen K, et al. AG-221, a first-in-class therapy targeting acute myeloid leukemia harboring oncogenic IDH2 mutations. Cancer Discov. 2017;7(5):478-493.

44. Brooks N, et al. Identification and characterization of LY3410738, a novel covalent inhibitor of cancer-associated mutant isocitrate dehydrogenase 1 (IDH1). Cancer Res. 2019;79(13 suppl):Abstract LB-274.

45. Nakagawa $\mathrm{M}$, et al. Selective inhibition of mutant IDH1 by DS-1001b ameliorates aberrant histone modifications and impairs tumor activity in chondrosarcoma. Oncogene. 2019;38(42):6835-6849.

46. Caravella JA, et al. Structure-based design and identification of FT-2102 (olutasidenib), a potent mutant-selective IDH1 inhibitor. J Med Chem. 2020;63(4):1612-1623.

47. Konteatis Z, et al. Vorasidenib (AG-881): a firstin-class, brain-penetrant dual inhibitor of mutant IDH1 and 2 for treatment of glioma. ACS Med Chem Lett. 2020;11(2):101-107.

48. Study of LY3410738 administered to patients with advanced solid tumors with IDH1 mutations. https://ClinicalTrials.gov/show/ NCT04521686. Updated August 11, 2021. Accessed September 22, 2021.

49. Study of DS-1001b in patients with gene IDH1-mutated gliomas. https://ClinicalTrials. gov/show/NCT03030066. Updated March 15, 2021. Accessed September 22, 2021.

50. A study of DS-1001b in patients with chemotherapy- and radiotherapy-naive IDH1 mutated WHO grade II glioma. https://ClinicalTrials.gov/ show/NCT04458272. Updated February 1, 2021. Accessed September 22, 2021.

51. Study of AG-120 and AG-881 in subjects with low grade glioma. https://ClinicalTrials.gov/ show/NCT03343197. Updated August 24, 2021. Accessed September 22, 2021.

52. Study of vorasidenib (AG-881) in participants with residual or recurrent grade 2 glioma with an IDH1 or IDH2 mutation (INDIGO). https://ClinicalTrials.gov/show/NCT04164901. Updated June 25, 2021. Accessed September 22, 2021.

53. Study of orally administered AG-881 in patients with advanced solid tumors, including gliomas, with an IDH1 and/or IDH2 mutation. https:// ClinicalTrials.gov/show/NCT02481154. Updated August 24, 2021. Accessed September 22, 2021.

54. DiNardo CD, et al. Mutant isocitrate dehydrogenase 1 inhibitor ivosidenib in combination with azacitidine for newly diagnosed acute myeloid leukemia. JClin Oncol. 2021;39(1):57-65.

55. De La Fuente MI, et al. A phase Ib/II study of olutasidenib in patients with relapsed/refractory IDH1 mutant gliomas: safety and efficacy as single agent and in combination with azacitidine. JClin Oncol. 2020;38(15 suppl):2505.

56. Kadiyala P, et al. Inhibition of 2-hydroxyglutarate elicits metabolic reprogramming and mutant IDH1 glioma immunity in mice. JClin Invest. 2021;131(4):139542.

57. A study of FT 2102 in participants with advanced solid tumors and gliomas with an IDH1 mutation. https://ClinicalTrials.gov/ show/NCT03684811. Updated March 29, 2021. Accessed September 22, 2021.

58. Gross MI, et al. Antitumor activity of the glutaminase inhibitor CB-839 in triple-negative breast cancer. Mol Cancer Ther. 2014;13(4):890-901.

59. Gross M, et al. Glutaminase inhibition with CB-839 enhances anti-tumor activity of PD-1 and PD-L1 antibodies by overcoming a metabolic checkpoint blocking T cell activation. Cancer Res.
2016;76(14 suppl):Abstract 2329.

60. Varghese S, et al. The glutaminase inhibitor CB-839 (telaglenastat) enhances the antimelanoma activity of T-cell-mediated immunotherapies. Mol Cancer Ther. 2021;20(3):500-511.

61. Caiola E, et al. Glutaminase inhibition on NSCLC depends on extracellular alanine exploitation. Cells. 2020;9(8):1766.

62. Matre $P$, et al. Inhibiting glutaminase in acute myeloid leukemia: metabolic dependency of selected AML subtypes. Oncotarget. 2016;7(48):79722-79735.

63. Raczka AM, Reynolds PA. Glutaminase inhibition in renal cell carcinoma therapy. Cancer Drug Resist. 2019;2(2):356-364.

64. Hoerner CR, et al. The 'Achilles heel' of metabolism in renal cell carcinoma: glutaminase inhibition as a rational treatment strategy. Kidney Cancer. 2019;3(1):15-29.

65. Reis L, et al. Dual inhibition of glutaminase and carnitine palmitoyltransferase decreases growth and migration of glutaminase inhibition-resistant triple-negative breast cancer cells. J Biol Chem. 2019;294(24):9342-9357.

66. Wang ES, et al. Phase 1 study of CB-839, a first-in-class, orally administered small molecule inhibitor of glutaminase in patients with relapsed/refractory leukemia. Blood. 2015;126(23):2566.

67. Novel PET/CT imaging biomarkers of CB-839 in combination with panitumumab and irinotecan in patients with metastatic and refractory RAS wildtype colorectal cancer. https://ClinicalTrials. gov/show/NCT03263429. Updated March 12, 2021. Accessed September 22, 2021.

68. CANTATA: CB-839 with cabozantinib vs. cabozantinib with placebo in patients with metastatic renal cell carcinoma. https://ClinicalTrials. gov/show/NCT03428217. Updated August 23, 2021. Accessed September 22, 2021.

69. Tannir NM, et al. CANTATA: primary analysis of a global, randomized, placebo (Pbo)-controlled, double-blind trial of telaglenastat (CB-839) + cabozantinib versus Pbo + cabozantinib in advanced/metastatic renal cell carcinoma (mRCC) patients (pts) who progressed on immune checkpoint inhibitor (ICI) or antiangiogenic therapies. J Clin Oncol. 2021;39(15 suppl):4501.

70. CB-839 $\mathrm{HCl}$ in combination with carfilzomib and dexamethasone in treating patients with recurrent or refractory multiple myeloma. https://ClinicalTrials.gov/show/NCT03798678. Updated August 26, 2021. Accessed September 22, 2021.

71. CB-839 in combination with niraparib in platinum resistant BRCA-wild-type ovarian cancer patients. https://ClinicalTrials.gov/show/ NCT03944902. Updated August 16, 2021. Accessed September 22, 2021.

72. A study of telaglenastat (CB-839) in combination with palbociclib in patients with solid tumors. https://ClinicalTrials.gov/show/ NCT03965845. Updated August 11, 2021. Accessed September 22, 2021.

73. Testing of the anti cancer drugs $\mathrm{CB}-839 \mathrm{HCl}$ (telaglenastat) and MLNO128 (sapanisertib) in advanced stage non-small cell lung cancer. https://ClinicalTrials.gov/show/ 
NCT04250545. Updated September 1, 2021. Accessed September 22, 2021.

74. Testing whether cancers with specific mutations respond better to glutaminase inhibitor, telaglenastat hydrochloride, anti-cancer treatment, BeGIN Study. https://ClinicalTrials.gov/show/ NCT03872427. Updated June 7, 2021. Accessed September 22, 2021.

75. Galan-Cobo A, et al. LKB1 and KEAP1/NRF2 pathways cooperatively promote metabolic reprogramming with enhanced glutamine dependence in KRAS-mutant lung adenocarcinoma. Cancer Res. 2019;79(13):3251-3267.

76. CB-839 with radiation therapy and temozolomide in treating patients with IDH-mutated diffuse astrocytoma or anaplastic astrocytoma. https://ClinicalTrials.gov/show/NCT03528642. Updated September 13, 2021. Accessed September 22, 2021.

77. Telaglenastat hydrochloride and osimertinib in treating patients with EGFR-mutated stage IV non-small cell lung cancer. https://ClinicalTrials. gov/show/NCT03831932. Updated September 2, 2021. Accessed September 22, 2021.

78. KEAPSAKE: a study of telaglenastat (CB-839) with standard-of-care chemoimmunotherapy in 1L KEAP1/NRF2-mutated, nonsquamous NSCLC. https://ClinicalTrials.gov/show/ NCT04265534. Updated September 2, 2021. Accessed September 22, 2021.

79. Dion HW, et al. 6-Diazo-5-oxo-L-norleucine, a new tumor-inhibitory substance. II. Isolation and characterization. JAm Chem Soc. 1956;78(13):3075-3077.

80. Lemberg KM, et al. We're not “DON” yet: optimal dosing and prodrug delivery of 6-diazo-5-oxo-L-norleucine. Mol Cancer Ther. 2018;17(9):1824-1832.

81. McGill G, et al. Pharmacological and initial therapeutic observations on 6-diazo-5-oxo-1-norleucine (DON) in human neoplastic disease. Cancer. 1957;10(6):1138-1150.

82. Rao R, Samak G. Role of glutamine in protection of intestinal epithelial tight junctions. J Epithel Biol Pharmacol. 2012;5(suppl 1-m7):47-54.

83. Tenora L, et al. Tumor-targeted delivery of 6-diazo-5-oxo-1-norleucine (DON) using substituted acetylated lysine prodrugs. J Med Chem. 2019;62(7):3524-3538.

84. Leone RD, et al. Glutamine blockade induces divergent metabolic programs to overcome tumor immune evasion. Science. 2019;366(6468):1013-1021.

85. Oh MH, et al. Targeting glutamine metabolism enhances tumor-specific immunity by modulating suppressive myeloid cells. JClin Invest. 2020;130(7):3865-3884.

86. Yokoyama Y, Wild R. Broad acting glutamine antagonism remodels the tumor microenvironment, induces distinctive immune modulation, and synergizes with immune checkpoint blockade. Cancer Res. 2020;80(16 suppl):Abstract 5607.

87. No authors listed. 34th Annual Meeting \& Pre-Conference Programs of the Society for Immunotherapy of Cancer (SITC 2019): part 1: National Harbor, MD, USA. 6-10 November 2019. JImmunother Cancer. 2019;7(suppl 1):282. 88. LeBoeuf SE, et al. Uncovering metabolic bottle- necks in KEAP1 mutant lung cancer. Cancer Res. 2020;80 (16 suppl):Abstract 2569.

89. Romero R, et al. Keap1 loss promotes Kras-driven lung cancer and results in dependence on glutaminolysis. Nat Med. 2017;23(11):1362-1368.

90. First-in-human study of DRP-104 (sirpiglenastat) as single agent and in combination with atezolizumab in patients with advanced solid tumors. https://ClinicalTrials.gov/ show/NCT04471415. Updated June 22, 2021. Accessed September 22, 2021.

91. Curtis NJ, et al. Pre-clinical pharmacology of AZD3965, a selective inhibitor of MCT1: DLBCL, NHL and Burkitt's lymphoma anti-tumor activity. Oncotarget. 2017;8(41):69219-69236.

92. Polański R, et al. Activity of the monocarboxylate transporter 1 inhibitor AZD3965 in small cell lung cancer. Clin Cancer Res. 2014;20(4):926-937.

93. Beloueche-Babari M, et al. Monocarboxylate transporter 1 blockade with AZD3965 inhibits lipid biosynthesis and increases tumour immune cell infiltration. Br J Cancer. 2020;122(6):895-903.

94. Lamb R, et al. Mitochondria as new therapeutic targets for eradicating cancer stem cells: quantitative proteomics and functional validation via MCT1/2 inhibition. Oncotarget. 2014;5(22):11029-11037.

95. A phase I trial of AZD3965 in patients with advanced cancer. https://ClinicalTrials.gov/ show/NCT01791595. Updated August 17, 2021. Accessed September 22, 2021.

96. McNeillis R, et al. A case of malignant hyperlactaemic acidosis appearing upon treatment with the mono-carboxylase transporter 1 inhibitor AZD3965. Br J Cancer. 2020;122(8):1141-1145.

97. van der Windt GJ, et al. Mitochondrial respiratory capacity is a critical regulator of CD8+ T cell memory development. Immunity. 2012;36(1):68-78.

98. Angelin A, et al. Foxp3 reprograms T cell metabolism to function in low-glucose, high-lactate environments. Cell Metab. 2017;25(6):1282-1293.

99. Scharping NE, et al. The tumor microenvironment represses $\mathrm{T}$ cell mitochondrial biogenesis to drive intratumoral $\mathrm{T}$ cell metabolic insufficiency and dysfunction. Immunity. 2016;45(2):374-388.

100.Zachar Z, et al. Non-redox-active lipoate derivates disrupt cancer cell mitochondrial metabolism and are potent anticancer agents in vivo. JMol Med (Berl). 2011;89(11):1137-1148.

101. Bingham PM, et al. Lipoic acid and lipoic acid analogs in cancer metabolism and chemotherapy. Expert Rev Clin Pharmacol. 2014;7(6):837-846.

102. Stuart SD, et al. A strategically designed small molecule attacks alpha-ketoglutarate dehydrogenase in tumor cells through a redox process. Cancer Metab. 2014;2(1):4

103. Egawa Y, et al. Therapeutic potential of CPI-613 for targeting tumorous mitochondrial energy metabolism and inhibiting autophagy in clear cell sarcoma. PLoS One. 2018;13(6):e0198940.

104. Rabben HL, et al. Neural signaling modulates metabolism of gastric cancer. iscience. 2021;24(2):102091.

105. Alistar A, et al. Safety and tolerability of the firstin-class agent CPI-613 in combination with modified FOLFIRINOX in patients with metastatic pancreatic cancer: a single-centre, open-label, dose-escalation, phase 1 trial. Lancet Oncol.
2017;18(6):770-778.

106.Philip PA, et al. A phase III open-label trial to evaluate efficacy and safety of CPI- 613 plus modified FOLFIRINOX ( $\mathrm{mFFX}$ ) versus FOLFIRINOX (FFX) in patients with metastatic adenocarcinoma of the pancreas. Future Oncol. 2019;15(28):3189-3196.

107. Pardee TS, et al. Devimistat in combination with high dose cytarabine and mitoxantrone compared with high dose cytarabine and mitoxantrone in older patients with relapsed/refractory acute myeloid leukemia: ARMADA 2000 Phase III study. Future Oncol. 2019;15(28):3197-3208.

108. Study evaluating efficacy and safety of FFX versus combination of CPI-613 with mFFX in patients with metastatic adenocarcinoma of the pancreas. https://ClinicalTrials.gov/show/ NCT03504423. Updated September 16, 2021. Accessed September 23, 2021.

109. Study evaluating efficacy and safety of CPI-613 in combination with HD cytarabine and mitoxantrone compared to HD cytarabine and mitoxantrone and control sub-groups: MEC and FLAG in older patients with R/R AML. https://ClinicalTrials.gov/show/NCT03504410. Updated September 16, 2021. Accessed September 23, 2021.

110. Molina JR, et al. An inhibitor of oxidative phosphorylation exploits cancer vulnerability. Nat Med. 2018;24(7):1036-1046.

111. Fischer GM, et al. Molecular profiling reveals unique immune and metabolic features of melanoma brain metastases. Cancer Discov. 2019;9(5):628-645.

112. Chen D, et al. Combination treatment with radiotherapy and a novel oxidative phosphorylation inhibitor overcomes PD-1 resistance and enhances antitumor immunity. JImmunother Cancer. 2020;8(1):e000289.

113. Yap TA, et al. Phase I trial of IACS-010759 (IACS), a potent, selective inhibitor of complex I of the mitochondrial electron transport chain, in patients (pts) with advanced solid tumors. JClin Oncol. 2019;37(15 suppl):3014.

114. Oxidative phosphorylation inhibitor IACS010759 in treating patients with relapsed or refractory acute myeloid leukemia. https://ClinicalTrials.gov/show/NCT02882321. Updated July 7,2020. Accessed September 23, 2021.

115. Rabinovich S, et al. Diversion of aspartate in ASS1-deficient tumours fosters de novo pyrimidine synthesis. Nature. 2015;527(7578):379-383.

116. Prudner BC, et al. Arginine starvation and docetaxel induce c-Myc-driven hENT1 surface expression to overcome gemcitabine resistance in ASS1-negative tumors. Clin Cancer Res. 2019;25(16):5122-5134.

117. Rodriguez PC, et al. Arginase I production in the tumor microenvironment by mature myeloid cells inhibits T-cell receptor expression and antigen-specific T-cell responses. Cancer Res. 2004;64(16):5839-5849.

118. A phase $1 / 2$ study of INCB001158 in combination with chemotherapy in subjects with solid tumors. https://ClinicalTrials.gov/ show/NCT03314935. Updated July 20, 2020. Accessed September 23, 2021.

119. Javle MM, et al. A phase I/II study of safety and efficacy of the arginase inhibitor INCBO01158 
plus chemotherapy in patients (Pts) with advanced biliary tract cancers. JClin Oncol. 2021;39(3 suppl):311.

120. Steggerda SM, et al. Inhibition of arginase by CB-1158 blocks myeloid cell-mediated immune suppression in the tumor microenvironment. J Immunother Cancer. 2017;5(1):101.

121. Arginase inhibitor INCBO01158 as a single agent and in combination with immune checkpoint therapy in patients with advanced/metastatic solid tumors. https://ClinicalTrials.gov/show/ NCT02903914. Updated August 30, 2021. Accessed September 23, 2021.

122. Papadopoulos KP, et al. CX-1158-101: a firstin-human phase 1 study of CB-1158, a small molecule inhibitor of arginase, as monotherapy and in combination with an anti-PD-1 checkpoint inhibitor in patients (pts) with solid tumors. JClin Oncol. 2017;35(15 suppl):3005.

123. Stega J, et al. A first-in-human study of the novel metabolism-based anti-cancer agent SM-88 in subjects with advanced metastatic cancer. Invest New Drugs. 2020;38(2):392-401.

124. Yin L, et al. Human MUC1 carcinoma antigen regulates intracellular oxidant levels and the apoptotic response to oxidative stress. J Biol Chem. 2003;278(37):35458-35464.

125. Vandell A, et al. In vitro and in vivo anticancer effects of D/L-alpha-metyrosine (SM-88), a novel metabolism-based therapy. Cancer Res. 2020;80(16 suppl):Abstract 5998.

126. Gartrell BA, et al. Phase II trial of SM-88, a cancer metabolism based therapy, in non-metastatic biochemical recurrent prostate cancer. Invest New Drugs. 2021;39(2):499-508.

127. Ocean A, et al. Phase II monotherapy efficacy of cancer metabolism targeting SM-88 in heavily pre-treated PDAC patients [abstract 719P]. Ann Oncol. 2019;30:v275.

128. Noel MS, et al. Phase II trial of SM-88 in patients with metastatic pancreatic cancer: preliminary results of the first stage. JClin Oncol. 2019;37(4 suppl):200.

129. Lu X. The role of large neutral amino acid transporter (LAT1) in cancer. Curr Cancer Drug Targets. 2019;19(11):863-876.

130. Higuchi K, et al. Characterization of the expression of LAT1 as a prognostic indicator and a therapeutic target in renal cell carcinoma. Sci Rep. 2019;9(1):16776.

131. Kim SG, et al. Differential expression and functional characterization of system $\mathrm{L}$ amino acid transporters in human normal osteoblast cells and osteogenic sarcoma cells. Anticancer Res. 2006;26(3a):1989-1996

132. Oda K, et al. L-type amino acid transporter 1 inhibitors inhibit tumor cell growth. Cancer Sci. 2010;101(1):173-179.

133. Yothaisong S, et al. Inhibition of L-type amino acid transporter 1 activity as a new therapeutic target for cholangiocarcinoma treatment. Tumour Biol. 2017;39(3):1010428317694545.

134. Werner A, et al. Reconstitution of $\mathrm{T}$ cell proliferation under arginine limitation: activated human T cells take up citrulline via L-type amino acid transporter 1 and use it to regenerate arginine after induction of argininosuccinate synthase expression. Front Immunol. 2017;8:864.
135. Okano N, et al. First-in-human phase I study of JPH203, an L-type amino acid transporter 1 inhibitor, in patients with advanced solid tumors. Invest New Drugs. 2020;38(5):1495-1506.

136. Okano N, et al. Biomarker analyses in patients with advanced solid tumors treated with the LAT1 inhibitor JPH203. In Vivo. 2020;34(5):2595-2606.

137. Konteatis Z, et al. Discovery of AG-270, a first-inclass oral MAT2A inhibitor for the treatment of tumors with homozygous MTAP deletion. JMed Chem. 2021;64(8):4430-4449.

138. Kalev P, et al. MAT2A inhibition blocks the growth of MTAP-deleted cancer cells by reducing PRMT5-dependent mRNA splicing and inducing DNA damage. Cancer Cell. 2021;39(2):209-224.

139. Heist RS, et al. A phase 1 trial of AG-270 in patients with advanced solid tumors or lymphoma with homozygous MTAP deletion. Mol Cancer Ther. 2019;18(12 suppl):Abstract PR03.

140. Kuhajda FP, et al. Fatty acid synthesis: a potential selective target for antineoplastic therapy. Proc Natl Acad Sci U S A. 1994;91(14):6379-6383.

141. Heuer TS, et al. FASN inhibition studies in preclinical tumor models identify biomarkers that align with in vitro and in vivo sensitivity to TVB-2640. Mol Cancer Ther. 2015;14(12 suppl 2):Abstract C175.

142. Heuer TS, et al. Preclinical studies characterize tumor type sensitivity to FASN inhibition and the mechanism and efficacy of novel drug combinations with TVB-2640. Cancer Res. 2016;76(14 suppl):Abstract 4743.

143. Ventura R, et al. Inhibition of de novo palmitate synthesis by fatty acid synthase induces apoptosis in tumor cells by remodeling cell membranes, inhibiting signaling pathways, and reprogramming gene expression. EBioMedicine. 2015;2(8):808-824.

144. Heuer TS, et al. FASN inhibition and taxane treatment combine to enhance anti-tumor efficacy in diverse xenograft tumor models through disruption of tubulin palmitoylation and microtubule organization and FASN inhibition-mediated effects on oncogenic signaling and gene expression. EBioMedicine. 2017;16:51-62.

145. Falchook G, et al. First-in-human study of the safety, pharmacokinetics, and pharmacodynamics of first-in-class fatty acid synthase inhibitor TVB-2640 alone and with a taxane in advanced tumors. EClinicalMedicine. 2021;34:100797.

146.TVB- 2640 in combination with bevacizum$a b$ in patients with first relapse of high grade astrocytoma. https://ClinicalTrials.gov/show/ NCT03032484. Updated April 28, 2020. Accessed September 23, 2021.

147. FASN inhibitor TVB-2640, paclitaxel, and trastuzumab in treating patients with HER2 positive advanced breast cancer. https://ClinicalTrials. gov/show/NCT03179904. Updated August 27, 2021. Accessed September 23, 2021.

148.Phase 2 study of TVB-2640 in KRAS non-small cell lung carcinomas. https://ClinicalTrials.gov/ show/NCT03808558. Updated February 23, 2021. Accessed September 23, 2021.

149. Lim SA, et al. Lipid signalling enforces functional specialization of $\mathrm{T}_{\mathrm{reg}}$ cells in tumours. Nature. 2021;591(7849):306-311
150. French KJ, et al. Pharmacology and antitumor activity of ABC294640, a selective inhibitor of sphingosine kinase-2. J Pharmacol Exp Ther. 2010;333(1):129-139.

151. Weigert A, et al. Sphingosine kinase 2 is a negative regulator of inflammatory macrophage activation. Biochim Biophys Acta Mol Cell Biol Lipids. 2019;1864(9):1235-1246.

152. Britten CD, et al. A phase I study of ABC294640, a first-in-class sphingosine kinase- 2 inhibitor, in patients with advanced solid tumors. Clin Cancer Res. 2017;23(16):4642-4650.

153. A study of ABC294640 (Yeliva $\mathbb{B}$ ) alone and in combination with hydroxychloroquine sulfate in treatment of patients with advanced cholangiocarcinoma. https://ClinicalTrials.gov/ show/NCT03377179. Updated March 18, 2021. Accessed September 23, 2021.

154. Addition of opaganib to androgen antagonists in patients with mCRPC. https://ClinicalTrials.gov/ show/NCT04207255. Updated July 30, 2021. Accessed September 23, 2021.

155. Heske CM. Beyond energy metabolism: exploiting the additional roles of NAMPT for cancer therapy. Front Oncol. 2019;9:1514.

156. Dasgupta A, et al. Targeting PAK4 inhibits Ras-mediated signaling and multiple oncogenic pathways in high-risk rhabdomyosarcoma. Cancer Res. 2021;81(1):199-212.

157. Abu Aboud O, et al. Dual and specific inhibition of NAMPT and PAK4 by KPT-9274 decreases kidney cancer growth. Mol Cancer Ther. 2016;15(9):2119-2129.

158. Li M, et al. Local targeting of NAD(+) salvage pathway alters the immune tumor microenvironment and enhances checkpoint immunotherapy in glioblastoma. Cancer Res. 2020;80(22):5024-5034.

159. Naing A, et al. A first in human phase 1 study of KPT-9274, a first in class dual inhibitor of PAK4 and NAMPT, in patients with advanced solid malignancies or NHL. Ann Oncol. 2017;28:v125.

160.PAK4 and NAMPT in patients with solid malignancies or NHL (PANAMA). https://ClinicalTrials.gov/show/NCT02702492. Updated June 25, 2021. Accessed September 23, 2021.

161. Cohen A, et al. PET imaging of glutamine metabolism in a clinical trial of metastatic colorectal cancer. J Nucl Med. 2020;61(suppl 1):630.

162. Nelson SJ, et al. Metabolic imaging of patients with prostate cancer using hyperpolarized [1-(1) (3)C]pyruvate. Sci Transl Med. 2013;5(198):198ra108.

163. Aggarwal R, et al. Hyperpolarized 1-[(13)C]pyruvate magnetic resonance imaging detects an early metabolic response to androgen ablation therapy in prostate cancer. Eur Urol. 2017;72(6):1028-1029.

164. Kurhanewicz J, et al. Hyperpolarized (13)C MRI: path to clinical translation in oncology. Neoplasia. 2019;21(1):1-16.

165. Pelletier JC, et al. Dipeptide prodrugs of the glutamate modulator riluzole. ACS Med Chem Lett. 2018;9(7):752-756.

166. Leone RD, Emens LA. Targeting adenosine for cancer immunotherapy. J Immunother Cancer. 2018;6(1):57.

167. Ensor CM, et al. Pegylated arginine deiminase (ADI-SS PEG20,000 mw) inhibits human mel- 
anomas and hepatocellular carcinomas in vitro and in vivo. Cancer Res. 2002;62(19):5443-5450.

168. Khan A, et al. Dual targeting of polyamine synthesis and uptake in diffuse intrinsic pontine gliomas. Nat Commun. 2021;12(1):971.

169. Guo B, et al. Antagonism of PPAR- $\gamma$ signaling expands human hematopoietic stem and progenitor cells by enhancing glycolysis. Nat Med. 2018;24(3):360-367.

170. Koblish HK, et al. Hydroxyamidine inhibitors of indoleamine-2,3-dioxygenase potently suppress systemic tryptophan catabolism and the growth of IDO-expressing tumors. Mol Cancer Ther. 2010;9(2):489-498.

171. Liu X, et al. Selective inhibition of IDO1 effectively regulates mediators of antitumor immunity. Blood. 2010;115(17):3520-3530.

172. Balog A, et al. Preclinical characterization of linrodostat mesylate, a novel, potent, and selective oral indoleamine 2,3-dioxygenase 1 inhibitor. Mol Cancer Ther. 2021;20(3):467-476.

173. Machida Y, et al. A potent blood-brain barrierpermeable mutant IDH1 inhibitor suppresses the growth of glioblastoma with IDH1 mutation in a patient-derived orthotopic xenograft model. $\mathrm{Mol}$ Cancer Ther. 2020;19(2):375-383.

174. Cho YS, et al. Discovery and evaluation of clinical candidate IDH305, a brain penetrant mutant IDH1 inhibitor. ACS Med Chem Lett. 2017;8(10):1116-1121.

175. Pardee TS, et al. A phase I study of CPI-613 in combination with high-dose cytarabine and mitoxantrone for relapsed or refractory acute myeloid leukemia. Clin Cancer Res. 2018;24(9):2060-2073.

176. Lee KC, et al. Translational assessment of mitochondrial dysfunction of pancreatic cancer from in vitro gene microarray and animal efficacy studies, to early clinical studies, via the novel tumor-specific anti-mitochondrial agent, CPI613. Ann Transl Med. 2014;2(9):91.

177. Vashisht Gopal YN, et al. A novel mitochondrial inhibitor blocks MAPK pathway and overcomes MAPK inhibitor resistance in melanoma. Clin Cancer Res. 2019;25(21):6429-6442.

178. Hafliger P, et al. The LAT1 inhibitor JPH2O3 reduces growth of thyroid carcinoma in a fully immunocompetent mouse model. J Exp Clin Cancer Res. 2018;37(1):234

179. Cibrian D, et al. Targeting L-type amino acid transporter 1 in innate and adaptive T cells efficiently controls skin inflammation. J Allergy Clin Immunol. 2020;145(1):199-214.

180. Hayashi K, et al. LAT1-specific inhibitor is effective against $\mathrm{T}$ cell-mediated allergic skin inflammation. Allergy. 2020;75(2):463-467.

181. Hung $\mathrm{MH}$, et al. Tumor methionine metabolism drives T-cell exhaustion in hepatocellular carcinoma. Nat Commun. 2021;12(1):1455

182.Dos Santos LM, et al. Methionine and methionine sulfoxide treatment induces M1/classical macrophage polarization and modulates oxidative stress and purinergic signaling parameters. $\mathrm{Mol}$ Cell Biochem. 2017;424(1-2):69-78.

183. Lewis CS, et al. Targeting sphingosine kinases for the treatment of cancer. In: Chalfant CE, Fisher PB, eds. Advances in Cancer Research. Academic Press; 2018:295-325.
184.Pembrolizumab (MK-3475) plus epacadostat vs standard of care in mRCC (KEYNOTE-679/ ECHO-302). https:/ClinicalTrials.gov/show/ NCT03260894. Updated September 1, 2021. Accessed September 23, 2021.

185. Pembrolizumab plus epacadostat, pembrolizumab monotherapy, and the EXTREME regimen in recurrent or metastatic head and neck squamous cell carcinoma (KEYNOTE-669/ ECHO-304). https://ClinicalTrials.gov/show/ NCT03358472. Updated September 22, 2021 Accessed September 23, 2021.

186. Tavo with electroporation, pembrolizumab, and epacadostat in patients with unresectable head and neck cancer. https://ClinicalTrials.gov/ show/NCT03823131. Updated August 9, 2021. Accessed September 23, 2021.

187. Epacadostat, pembrolizumab, and CRS-207, with or without CY/GVAX pancreas in patients with metastatic pancreas cancer. https://ClinicalTrials.gov/show/NCT03006302. August 20, 2021. Accessed September 23, 2021.

188. A study of epacadostat, an IDO1 inhibitor, in combination with pembrolizumab in patients with metastatic and/or locally advanced sarcoma. https://ClinicalTrials.gov/show/ NCT03414229. Updated April 21, 2021. Accessed September 23, 2021

189. INCMGA00012 and epacadostat in combination with radiation and bevacizumab in patients with recurrent gliomas. https:/ClinicalTrials.gov/ show/NCT03532295. Updated September 16, 2021. Accessed September 23, 2021.

190. An umbrella study to determine the safety and efficacy of various monotherapy or combination therapies in neoadjuvant urothelial carcinoma. https:// ClinicalTrials.gov/show/NCT04586244. Updated July 2, 2021. Accessed September 23, 2021.

191. UCDCC\#271: phase I/II trial of epacadostat, intralesional SD101, radiotherapy in patients with lymphoma. https://ClinicalTrials.gov/ show/NCT03322384. Updated October 8, 2020. Accessed September 23, 2021.

192.DEC-205/NY-ESO-1 fusion protein CDX-1401, poly ICLC, and IDO1 inhibitor INCBO24360 in treating patients with ovarian, fallopian tube, or primary peritoneal cancer in remission. https:// ClinicalTrials.gov/show/NCT02166905. Updated July 22, 2021. Accessed September 23, 2021.

193. Combination study of SV-BR-1-GM in combination with INCMGA00012 and epacadostat. https:// ClinicalTrials.gov/show/NCT03328026. Updated September 16, 2021. Accessed September 23, 2021.

194.Safety and efficacy of retifanlimab (INCMGA00012) alone or in combination with other therapies in participants with advanced or metastatic endometrial cancer who have progressed on or after platinum-based chemotherapy. https:// ClinicalTrials.gov/show/NCT04463771. Updated July 29, 2021. Accessed September 23, 2021

195. An investigational immuno-therapy study of BMS-986205 given in combination with nivolumab and in combination with both nivolumab and ipilimumab in cancers that are advanced or have spread. https://ClinicalTrials. gov/show/NCT02658890. Updated August 10, 2020. Accessed September 23, 2021.

196. A study of BMS-986205 alone and in combina- tion with nivolumab in Chinese patients with advanced malignant solid tumors. https://ClinicalTrials.gov/show/NCT03792750. Updated August 10, 2020. Accessed September 23, 2021.

197. An investigational study of immunotherapy combinations in participants with solid cancers that are advanced or have spread. https://ClinicalTrials.gov/show/NCT03459222. Updated October 22, 2020. Accessed September 23, 2021

198.A study to test combination treatments in patients with advanced gastric cancer. https:// ClinicalTrials.gov/show/NCT02935634. Updated July 19, 2021. Accessed September 23, 2021.

199. A study to test combination treatments in people with advanced renal cell carcinoma. https://ClinicalTrials.gov/show/NCT02996110. Updated June 17, 2021. Accessed September 23, 2021

200.Open-label study of FT-2102 with or without azacitidine or cytarabine in patients with AML or MDS with an IDH1 mutation. https://ClinicalTrials.gov/show/NCT02719574. Updated August 17, 2021. Accessed September 23, 2021.

201. Study of oral LY3410738 in patients with advanced hematologic malignancies with IDH1 or IDH2 mutations. https://ClinicalTrials.gov/ show/NCT04603001. Updated September 9, 2021. Accessed September 23, 2021.

202.A study of IDH305 in patients with advanced malignancies that harbor IDH1R132 mutations. https://ClinicalTrials.gov/show/ NCT02381886. Updated July 26, 2021. Accessed September 23, 2021.

203. Telaglenastat + talazoparib in prostate cancer. https://ClinicalTrials.gov/show/ NCT04824937. Updated April 1, 2021. Accessed September 23, 2021.

204.Glutaminase inhibitor CB-839 and azacitidine in treating patients with advanced myelodysplastic syndrome. https://ClinicalTrials.gov/ show/NCT03047993. Updated August 30, 2021. Accessed September 23, 2021

205. CPI-613 in combination with bendamustine in patients with relapsed/refractory T-cell non-Hodgkin lymphoma. https://ClinicalTrials. gov/show/NCT04217317. Updated November 17, 2020. Accessed September 23, 2021.

206.A study of CPI- 613 for patients with relapsed or refractory Burkitt lymphoma/leukemia or high-grade B-cell lymphoma with high-risk translocations. https://ClinicalTrials.gov/show/ NCT03793140. Updated November 18, 2020. Accessed September 23, 2021

207. CPI-613 in combination with modified FOLFIRINOX in locally advanced pancreatic cancer. https://ClinicalTrials.gov/show/ NCT03699319. Updated January 8, 2021. Accessed September 23, 2021.

208.CPI-613, cytarabine, and mitoxantrone hydrochloride in treating patients with relapsed or refractory acute myeloid leukemia or granulocytic sarcoma. https://ClinicalTrials.gov/show/ NCT02484391. Updated October 23, 2020. Accessed September 23, 2021

209. To evaluate maximally tolerated dose (MTD), safety and efficacy of CPI- $613^{\circledR}$ (devimistat) plus hydroxychloroquine in patients with relapsed or refractory clear cell sarcoma of soft tissue. https://ClinicalTrials.gov/show/NCT04593758. 
Updated September 22, 2021. Accessed September 23, 2021.

210. Gemcitabine and cisplatin with or without CPI-613 as first line therapy for patients with advanced unresectable biliary tract cancer (BilT-04). https://ClinicalTrials.gov/show/ NCT04203160. Updated June 4, 2021. Accessed September 23, 2021.

211. INCB001158 combined with subcutaneous (SC) daratumumab, compared to daratumumab SC, in relapsed or refractory multiple myeloma. https:// ClinicalTrials.gov/show/NCT03837509. Updated July 2, 2021. Accessed September 23, 2021.

212. A study of INCMGA00012, INCB001158, and the combination in Japanese participants with advanced solid tumors. https://ClinicalTrials. gov/show/NCT03910530. Updated June 16, 2021. Accessed September 23, 2021.

213. A multi-center trial to evaluate multiple regimens in metastatic pancreatic cancer. https://Clinical-
Trials.gov/show/NCT04229004. Updated June 16, 2021. Accessed September 23, 2021.

214. Study of SM-88 in advanced cancers. https:// ClinicalTrials.gov/show/NCT02562612. Updated March 8, 2021. Accessed September 23, 2021. 215. Oral SM-88 in patients with metastatic $\mathrm{HR}+$ / HER2- breast cancer. https://ClinicalTrials.gov/ show/NCT04720664. Updated September 8, 2021. Accessed September 23, 2021.

216. A randomized phase $2 / 3$ multi-center study of SM- 88 in patients with metastatic pancreatic cancer. https://ClinicalTrials.gov/show/ NCT03512756. Updated June 21, 2021. Accessed September 23, 2021.

217. SM-88 as maintenance therapy for advanced Ewing's sarcoma patients and as salvage therapy for sarcoma patients. https://ClinicalTrials.gov/ show/NCT03778996. Updated April 1, 2021. Accessed September 23, 2021.

218.University Hospital Medical Information Net- work Clinical Trials Registry (UMIN-CTR). Exploratory analyses of biomarker using change of blood free amino-acid concentration related to JPH2O3(LAT1 inhibitor). https://upload.umin. ac.jp/cgi-open-bin/ctr_e/ctr_view.cgi?recptno= R000019508. Updated September 7, 2020. Accessed September 23, 2021.

219. Study of AG-270 in participants with advanced solid tumors or lymphoma with MTAP loss. https:// ClinicalTrials.gov/show/NCT03435250. Updated August 19, 2021. Accessed September 23, 2021.

220.TVB 2640 for resectable colon cancer other resectable cancers; a window trial. https://ClinicalTrials.gov/show/NCT02980029. Updated April 19, 2021. Accessed September 23, 2021.

221. A study of evaluating dual inhibitor of PAK 4 and NAMPT ATG-019 in advanced solid tumors or non-Hodgkin's lymphoma. https://ClinicalTrials. gov/show/NCT04281420. Updated May 7, 2021. Accessed September 23, 2021. 